\title{
FRACTIONAL RANDOM FIELDS ASSOCIATED WITH STOCHASTIC FRACTIONAL HEAT EQUATIONS
}

\author{
M. YA. KELBERT,* University of Wales Swansea \\ N. N. LEONENKO, ${ }^{* *}$ Cardiff University \\ M. D. RUIZ-MEDINA, ${ }^{* * *}$ University of Granada
}

\begin{abstract}
This paper introduces a convenient class of spatiotemporal random field models that can be interpreted as the mean-square solutions of stochastic fractional evolution equations.

Keywords: Fractional random field; operator spectral method; fractional heat equation; spatiotemporal spectral density; Matérn class; geostatistics
\end{abstract}

2000 Mathematics Subject Classification: Primary 60G60; 60G20

\section{Introduction}

Recent studies have found that data in many fields of application (including geostatistics, hydrology, turbulence, economics, and finance) display fractal structure (see Adler (1981), Hosking (1981), Chambers (1996), Woyczyński (1998), Hilfer (2000), Christakos (2000), and the references therein).

Fractional operators are the natural mathematical objects to describe fractal phenomena. Our study is motivated by a paper of Gay and Heyde (1990), which introduced a class of random processes and fields involving both short- and long-range dependence via a stochastic differential equation with fractional differential operators, for which there is a unified approach to the corresponding parameter inference (see Heyde (1997)).

Earlier, Granger and Joyeux (1980) and Hosking (1981) constructed long-memory time series in discrete time via fractional differencing, and Chambers (1996) used fractional derivatives to obtain long-memory phenomena for continuous-time stochastic processes. Some other examples of fractional random fields can be found in Anh et al. (1999), Anh and Leonenko (2000), (2001), (2002) and Ruiz-Medina et al. (2001), (2003), (2004).

In fact, the approach used by Gay and Heyde (1990) has its origins in the classic paper by Whittle (1963), who derived the spectral densities and covariance functions of some spatial and spatiotemporal random fields. The goal of this paper is to employ the theory of generalized random fields on fractional Sobolev spaces, which was developed by Ruiz-Medina et al. (2001), (2004), to justify the approach of Gay and Heyde (1990) (in Section 2) and to introduce a new class of spatiotemporal random field models, which can be interpreted as the mean-square solutions of stochastic fractional evolution (or heat) equations (see Section 3).

Received 31 March 2003; revision received 28 May 2004.

* Postal address: Department of Mathematics, University of Wales Swansea, Singleton Park, Swansea SA2 8PP, UK.

** Postal address: Cardiff School of Mathematics, Cardiff University, Senghennydd Road, Cardiff CF24 4AG, UK.

Email address: leonenkon@ cardiff.ac.uk

*** Postal address: Department of Statistics and Operational Research, University of Granada, Campus Fuente Nueva s/n, E-18071 Granada, Spain. 
Christakos (2000, p. 225) also considered, in a geostatistical context, a model family related to the second-order definition of solutions to stochastic fractional evolution equations (see also Stein (1999)). Finally, we obtain in Section 4 the limiting distribution of polynomials of the rescaled solution to the stochastic fractional evolution equation studied in Section 3.

\section{A stochastic differential equation of Gay and Heyde} form

Firstly, we write the stochastic differential equation of Gay and Heyde (1990) in its heuristic

$$
(D+\alpha)^{v} X(t)=\varepsilon(t), \quad t \in \mathbb{R},
$$

in terms of the operator $D \equiv D_{t}=\mathrm{d} / \mathrm{d} t, v>0, \alpha \in \mathbb{R}$. Here, $\{\varepsilon(t), t \in \mathbb{R}\}$ is a zero-mean white noise, that is, a stochastic process satisfying

$$
\mathrm{E}[\varepsilon(f) \varepsilon(g)]=\sigma^{2}\langle f, g\rangle_{L^{2}(\mathbb{R})}, \quad \sigma^{2}>0,
$$

for $f, g \in L^{2}(\mathbb{R})$, the space of square-integrable functions. We write

$$
\varepsilon(f)=\int_{-\infty}^{\infty} f(t) \mathrm{d} Z_{\varepsilon}(t)
$$

with $Z_{\varepsilon}(t)$ being a process with orthogonal increments, defined by

$$
Z_{\varepsilon}(t)=\underset{n \rightarrow \infty}{\lim \text { in q.m. }} . \int_{0}^{t} X^{(n)}(s) \mathrm{d} s
$$

for $\left\{X^{(n)}(s), s \in \mathbb{R}\right\}, n \in \mathbf{N}$, a sequence of quadratic-mean (q.m.) continuous processes converging to a white noise (see, e.g. Wong and Hajek (1985, pp. 109-115)). The fractional operator in (2.1) is defined by the formal binomial expansion

$$
(D+\alpha)^{v}=\sum_{j=0}^{\infty}\left(\begin{array}{l}
v \\
j
\end{array}\right) D^{j} \alpha^{\nu-j},
$$

where the binomial coefficients are

$$
\left(\begin{array}{l}
v \\
j
\end{array}\right)=\frac{v(v-1) \cdots(v-j+1)}{j !}=\frac{(-1)^{j}(-v)_{j}}{j !},
$$

and the Pochhammer symbol

$$
(\lambda)_{j}= \begin{cases}1 & \text { (if } j=0) \\ \lambda(\lambda+1) \cdots(\lambda+j-1) & (\text { if } j=1,2, \ldots)\end{cases}
$$

is defined, in terms of the gamma function, as

$$
(\lambda)_{j}=\Gamma(\lambda+j) / \Gamma(\lambda), \quad \lambda \neq 0,-1,-2, \ldots .
$$

The formal second-order stationary solution to (2.1) has the spectral density

$$
f_{\alpha, v}^{X}(\lambda)=\frac{\sigma^{2}}{2 \pi} \frac{1}{\left(\lambda^{2}+\alpha^{2}\right)^{v}}, \quad \lambda \in \mathbb{R}, v>\frac{1}{2} .
$$


A second-order stationary stochastic process $X=\{X(t), t \in \mathbb{R}\}$ with the spectral density (2.3) exhibits short-range dependence if $\alpha \neq 0, v>\frac{1}{2}$; long-range dependence holds for $\alpha=0, v \in\left(0, \frac{1}{2}\right)$.

In fact, when $\alpha=0$ and $v \in\left(0, \frac{1}{2}\right),(2.3)$ is not the spectral density of a stationary process, but of a self-similar stationary increments process of order $\frac{1}{2}-v$ (see, e.g. Dobrushin (1979)). It is, however, very closely approximated by a (continuous) stationary process

$$
Z(t)=\int_{t-1 / 2}^{t+1 / 2} Y(s) \mathrm{d} s
$$

with spectral density

$$
f^{Z}(\lambda)=\frac{4 \sigma^{2}}{2 \pi} \frac{\sin ^{2}(\lambda / 2)}{\lambda^{2+2 v}}, \quad \lambda \in \mathbb{R}, 0<v<\frac{1}{2},
$$

where the process $Y=\{Y(t), t \in \mathbb{R}\}$ has stationary increments and spectral density (2.3) (see Gay and Heyde (1990) for details and generalizations).

Note that the covariance function

$$
B(x)=\int_{\mathbb{R}} \cos (\lambda x) \frac{\sigma^{2}}{2 \pi} \frac{1}{\left(\lambda^{2}+\alpha^{2}\right)^{v}} \mathrm{~d} \lambda, \quad x \in \mathbb{R}, v>\frac{1}{2},
$$

belongs to the Matérn class (see Stein (1999, pp. 31-32)) and takes the form

$$
B(x)=\frac{\sigma^{2}}{2 \pi} \frac{\sqrt{\pi}}{2^{v-1} \Gamma(\nu) \alpha^{2 v-1}} K_{\nu-1 / 2}(\alpha|x|)(\alpha|x|)^{\nu-1 / 2},
$$

where

$$
K_{\lambda}(y)=\int_{0}^{\infty} \mathrm{e}^{-y \cosh (u)} \cosh (\lambda u) \mathrm{d} u, \quad y>0,
$$

is the Macdonald function with index $\lambda \in R$.

Note that the definition (2.2) of a fractional differential operator is formal, since it is based on the binomial series

$$
(1+t)^{v}=\sum_{j=0}^{\infty}\left(\begin{array}{l}
v \\
j
\end{array}\right) t^{j}, \quad v>0,|t|<1 .
$$

If $v$ is neither a natural number nor zero, the series (2.4) converges under the conditions that either $|t|<1$; $t=1$ and $v>-1$; or $t=-1$ and $v>0$. A formal computation using (2.2), (2.4), and the hypergeometric series

$$
(1-t)^{-v}=\sum_{j=0}^{\infty}(v)_{j} \frac{t^{j}}{j !}, \quad v>0,|t|<1,
$$

which again has finite radius of convergence, leads to the spectral density (2.3).

The above approach has its origins in the famous paper by Whittle (1963) (see also Yadrenko (1983)).

Here, the approach of Gay and Heyde (1990) is justified using the theory of generalized random fields (GRFs) on fractional Sobolev spaces $H^{v}(\mathbb{R})$ and the concept of dual GRFs, 
which was developed by Ruiz-Medina et al. (2001), (2004). Basic facts on Sobolev spaces $H^{\nu}(\mathbb{R})$ of integer and fractional order $v$, and on the spectral theory of self-adjoint operators on a separable Hilbert space, can be found in Dunford and Schwartz (1971), Adams (1975), Triebel (1978), Dautray and Lions (1985a), (1985b), and Ramm (1990).

Let $\mathcal{L}$ be the self-adjoint operator $-\mathrm{i}(\mathrm{d} / \mathrm{d} t)$ on the separable Hilbert space $L^{2}(\mathbb{R})$ of complex-valued functions that are square-integrable on $\mathbb{R}$. The domain $\mathscr{D}(\mathcal{L})$ of $\mathcal{L}$ is dense in $L^{2}(\mathbb{R})$. Since $(D+\alpha)^{v}=(\mathrm{i} \mathcal{L}+\alpha)^{v},(D+\alpha)^{v}$ admits the following spectral representation (see Dautray and Lions (1985b, p. 140)): for $\varphi \in H^{\nu}(\mathbb{R})=\mathscr{D}\left((D+\alpha)^{\nu}\right)$ and $g \in L^{2}(\mathbb{R})$,

$$
(D+\alpha)^{v}(\varphi)(g)=\int_{\mathbb{R}} \int_{\mathbb{R}} \bar{g}(t)\left[\int_{\Lambda}(\mathrm{i} \lambda+\alpha)^{v} \Phi(t, s, \lambda) \mathrm{d} \lambda\right] \varphi(s) \mathrm{d} s \mathrm{~d} t,
$$

where (here and below) $\bar{g}(t)$ is the complex conjugate function, $\Phi(t, s, \lambda)=(2 \pi)^{-1} \times$ $\exp (\mathrm{i} \lambda(t-s))$ is the spectral kernel of the operator $\mathcal{L}, \mathrm{d} \lambda=\mathrm{d} \rho(\lambda)$ is the spectral measure of $\mathcal{L}$, and $\Lambda=\mathbb{R}$ is the continuous spectrum of $\mathcal{L}$. We also have, for $f, g \in L^{2}(\mathbb{R})$ and $h \in H^{-v}(\mathbb{R})=\mathscr{D}\left((D+\alpha)^{-v}\right)$,

$$
\begin{aligned}
\left((D+\alpha)^{v}\right)^{-1}(h)(f) & =\int_{\mathbb{R}} \int_{\mathbb{R}} \bar{f}(t)\left[\int_{\Lambda}(\mathrm{i} \lambda+\alpha)^{-v} \Phi(t, s, \lambda) \mathrm{d} \lambda\right] h(s) \mathrm{d} s \mathrm{~d} t \\
& =(D+\alpha)^{-v}(h)(f) \quad \text { for all } h \in \mathscr{D}\left((D+\alpha)^{-v}\right) \\
& =H^{-v}(\mathbb{R}), \\
\left((D+\alpha)^{v}\right)^{\dagger}(\varphi)(g) & =\int_{\mathbb{R}} \int_{\mathbb{R}} \bar{g}(t)\left[\int_{\Lambda}(-\mathrm{i} \lambda+\alpha)^{v} \Phi(t, s, \lambda) \mathrm{d} \lambda\right] \varphi(s) \mathrm{d} s \mathrm{~d} t \\
& =\left(D^{\dagger}+\alpha\right)^{v}(\varphi)(f) \quad \text { for all } \varphi \in \mathscr{D}\left(\left(D^{\dagger}+\alpha\right)^{v}\right) \\
& =H^{v}(\mathbb{R}), \\
{\left[\left((D+\alpha)^{v}\right)^{-1}\right]^{\dagger}(h)(f) } & =\int_{\mathbb{R}} \int_{\mathbb{R}} \bar{f}(t)\left[\int_{\Lambda}(-\mathrm{i} \lambda+\alpha)^{-v} \Phi(t, s, \lambda) \mathrm{d} \lambda\right] h(s) \mathrm{d} s \mathrm{~d} t \\
& =\left(D^{\dagger}+\alpha\right)^{-v}(h)(f) \quad \text { for all } h \in \mathscr{D}\left(\left(D^{\dagger}+\alpha\right)^{-v}\right) \\
& =H^{-v}(\mathbb{R}),
\end{aligned}
$$

where ' $\uparrow$ ' stands for the adjoint operator.

The mean-square (m.s.) solution to (2.1) is defined below using the theory of GRFs on fractional Sobolev spaces. We assume that $\sigma=1$, without loss of generality.

Theorem 2.1. Let $X_{-v}$ be a GRF defined, in the mean-square sense, as

$$
\begin{gathered}
X_{-v}(h) \stackrel{\text { m.s. }}{=} \int_{\mathbb{R}} \bar{h}(t) \int_{\mathbb{R}} l(t, s) \mathrm{d} \varepsilon(s) \mathrm{d} t \\
\stackrel{\text { m.s. }}{=} \int_{\mathbb{R}} \bar{h}(t) \mathcal{X}_{-v}(t) \mathrm{d} t
\end{gathered}
$$

for all $h \in L^{2}(\mathbb{R})$ and $v>0$, where $\varepsilon$ is the generalized white noise process appearing in (2.1). Here,

$$
\begin{aligned}
l(t, s) & =\int_{\Lambda}(\mathrm{i} \lambda+\alpha)^{-v} \Phi(t, s, \lambda) \mathrm{d} \lambda \\
& =(2 \pi)^{-1} \int_{\Lambda} \exp (\mathrm{i} \lambda(t-s))(\mathrm{i} \lambda+\alpha)^{-v} \mathrm{~d} \lambda .
\end{aligned}
$$


Then for $v \leq \frac{1}{2}, X_{-v}$ defines - in the weak sense-a mean-square solution $X_{-v}$ to (2.1) and, for $v>\frac{1}{2}$, defines - in the strong sense - the unique mean-square continuous solution $\mathcal{X}_{-v}$ to (2.1).

The GRF $X_{-v}$ has reproducing kernel Hilbert space $(R K H S)$ isomorphic to the space $H^{v}(\mathbb{R})$.

Remark 2.1. Note that, as $l$ is a deterministic function, the integral

$$
\int_{\mathbb{R}} l(t, s) \mathrm{d} \varepsilon(s)
$$

can be interpreted as a second-order stochastic integral (see, e.g. Wong and Hajek (1985, p. 141 and pp. 97-104)). Hence, as for $v>\frac{1}{2}$, the integral operator defined by $l$ is in the trace class, and so the above integral exists. For $v \leq \frac{1}{2}$, the integral is defined in the weak sense; that is, such an integral exists when we consider integration with a test function in the fractional Sobolev space $H^{-v}(\mathbb{R})$, to which the Dirac delta distribution does not belong: specifically,

$$
\int_{\mathbb{R}} \int_{\mathbb{R}}\left[\int_{\mathbb{R}} l(t, s) l(u, s) \mathrm{d} s\right] h(t) g(u) \mathrm{d} t \mathrm{~d} u<\infty,
$$

for $h, g \in H^{-v}(\mathbb{R})$.

Proof of Theorem 2.1. Using a version of the stochastic Fubuni theorem (cf. Protter (1990, Theorem 4.6) and Karatzas and Shreve (1991, p. 225)), we obtain for the GRF $X_{-v}$ defined in (2.5), and for $h \in L^{2}(\mathbb{R}) \subset H^{-v}(\mathbb{R})$, the following representation:

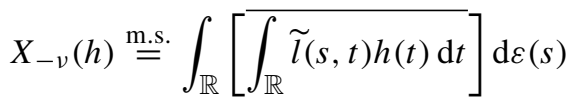

$$
\begin{aligned}
& \stackrel{\text { m.s. }}{=} \varepsilon\left(\left(D^{\dagger}+\alpha\right)^{-v} h\right) \text {. }
\end{aligned}
$$

Here we have used the facts that, for $v>\frac{1}{2}$, the above second-order integral exists, due to the regularity order of $l$ (related to the Hilbert-Schmidt property of the integral operator defined by $l$ ) and that, for $v \leq \frac{1}{2}$, the function $h$ allows the integral with respect to $\mathrm{d} \varepsilon$ to be defined (see Remark 2.1). Note that

$$
\widetilde{l}(s, t)=l(-s,-t)=(2 \pi)^{-1} \int_{\mathbb{R}} \exp (\mathrm{i} \lambda(s-t))(-\mathrm{i} \lambda+\alpha)^{-v} \mathrm{~d} \lambda .
$$

Therefore, for $h=\left(D^{\dagger}+\alpha\right)^{v} \varphi$ with $\varphi \in \mathscr{D}\left(\left(D^{\dagger}+\alpha\right)^{v}\right)=H^{v}(\mathbb{R})$,

$$
X_{-v}\left(\left(D^{\dagger}+\alpha\right)^{v} \varphi\right) \stackrel{\text { m.s. }}{=} \varepsilon\left(\left(D^{\dagger}+\alpha\right)^{-v}\left(D^{\dagger}+\alpha\right)^{v} \varphi\right) \stackrel{\text { m.s. }}{=} \varepsilon(\varphi),
$$

which means that

$$
\begin{aligned}
\int_{\mathbb{R}} \overline{\left(D^{\dagger}+\alpha\right)^{v} \varphi(t)} X_{-v}(t) \mathrm{d} t \stackrel{\text { m.s. }}{=} \int_{\mathbb{R}} \bar{\varphi}(t)(D+\alpha)^{v} \mathcal{X}_{-v}(t) \mathrm{d} t \\
\stackrel{\text { m.s. }}{=} \int_{\mathbb{R}} \bar{\varphi}(t) \mathrm{d} \varepsilon(t)
\end{aligned}
$$

or, equivalently, that $\mathcal{X}_{-v}$ defines - in the weak sense - a mean-square solution to (2.1). Here, as before, ' $\stackrel{\text { m.s., }}{=}$ stands for equality in the mean-square sense and, therefore, the integrals of random fields that have appeared up to this point are also defined in this sense. 
From embedding theorems between fractional Besov spaces (see Triebel (1978)), for $v>\frac{1}{2}$ the covariance function of $X_{-v}$ is continuous and $X_{-v}$ defines the unique mean-square continuous solution to (2.1).

The $(-v)$-GRF $X_{-v}$, defined on the space of test functions $H^{-v}(\mathbb{R})$ having weak-sense regularity order $-v$, , satisfies the duality condition introduced in Definition A.2 (see Appendix A), since the dual $\widetilde{X}_{-v}$ of $X_{-v}$ is defined as

$$
\tilde{X}_{-v}(\phi) \stackrel{\text { m.s. }}{=} \varepsilon\left((D+\alpha)^{v} \phi\right) \text { for all } \phi \in H^{v}(\mathbb{R}),
$$

where $\varepsilon$ is the generalized white noise defining (2.1). The equivalence between norms on the spaces $H^{v}(\mathbb{R})$ and $\mathscr{H}\left(X_{-v}\right)$ then follows from Proposition A.1 in Appendix A, with $\alpha=-v$.

Remark 2.2. As $L^{2}(\mathbb{R})$ is a dense subspace of the parameter space $H^{-v}(\mathbb{R})$ of the $(-v)$-GRF $X_{-v}$ defined above (see Appendix A), we use it in the proof of Theorem 2.1 to emphasize the weak-sense and strong-sense definitions of the random field $\mathcal{X}_{-v}$.

The covariance operator $R_{X_{-v}}$ of $X_{-v}$ is given by

$$
R_{X_{-v}}=(D+\alpha)^{-v}\left(D^{\dagger}+\alpha\right)^{-v} .
$$

Therefore, the following identities hold:

$$
\begin{aligned}
R_{X_{-v}}(f)(g) & =\int_{\Lambda}(\mathrm{i} \lambda+\alpha)^{-v}(-\mathrm{i} \lambda+\alpha)^{-v}\left(\mathrm{~d} E_{\lambda}(f), g\right) \\
& =\int_{\Lambda}\left(\lambda^{2}+\alpha^{2}\right)^{-v}\left(\mathrm{~d} E_{\lambda}(f), g\right) \text { for all } f, g \in L^{2}(\mathbb{R}),
\end{aligned}
$$

where $E_{\lambda}, \lambda \in \Lambda$, is the spectral family associated with the operator $\mathcal{L}$. The covariance kernel $B X_{-v}$ of $\mathcal{X}_{-v}$ then admits the following spectral representation, for $f, g \in L^{2}(\mathbb{R})$ :

$$
\begin{aligned}
\int_{\mathbb{R}} \int_{\mathbb{R}} \bar{g}(t) B_{X_{-v}}(t, s) f(s) \mathrm{d} s \mathrm{~d} t \\
\quad=\int_{\mathbb{R}} \int_{\mathbb{R}} \bar{g}(t)\left[(2 \pi)^{-1} \int_{\mathbb{R}}\left(\lambda^{2}+\alpha^{2}\right)^{-v} \exp (\mathrm{i} \lambda(t-s)) \mathrm{d} \lambda\right] f(s) \mathrm{d} s \mathrm{~d} t .
\end{aligned}
$$

Thus, the spectral density of the formal second-order solution to (2.1) is given by (2.3).

The fractional Hölder exponent (in the mean-square sense) and the sample-path fractional Hölder exponent (in the Gaussian case) of the mean-square solution to (2.1) are now studied. In the Gaussian case, we also provide the Hausdorff dimension $\operatorname{dim}_{H}$ of the image and graph of sample paths (see, e.g. Adler (1981, p. 204)). Let us assume that all random variables are defined on a complete probability space $(\Omega, \mathcal{F}, \mathrm{P})$.

Proposition 2.1. For $v>\frac{1}{2}$, the solution $\chi_{-v}$ to (2.1) is Hölder continuous, in the meansquare sense, of order $v-\frac{1}{2}$.

In the Gaussian case, the sample paths of $\mathcal{X}_{-v}$ have modulus of continuity $\Lambda(\delta)$ of order $\delta^{\nu-1 / 2}(|\ln \delta|)^{-1}$ and satisfy, with probability 1 ,

$$
\begin{aligned}
\operatorname{dim}_{H}\left(\operatorname{image}\left(X_{-v}(\cdot)\right)\right) & =1 \wedge\left(\frac{1}{v-\frac{1}{2}}\right), \\
\operatorname{dim}_{H}\left(\operatorname{graph}\left(X_{-v}(\cdot)\right)\right) & =\left(\frac{1}{v-\frac{1}{2}}\right) \wedge\left(2-\left(v-\frac{1}{2}\right)\right) .
\end{aligned}
$$


Remark 2.3. For $0<v-\frac{1}{2}<1$, the Hölder exponent of $X_{-v}$, in the mean-square sense, is between 0 and 1 . Hence, $X_{-v}$ is continuous in the mean-square sense but is not differentiable in this sense. We can refer to $\mathcal{X}_{-v}$ as a fractal random field in the mean-square sense.

Proof of Proposition 2.1. From Theorem 2.1,

$$
\begin{aligned}
\mathrm{E}\left|\mathcal{X}_{-v}(s+h)-X_{-v}(s)\right|^{2} & =2\left(B X_{-v}(0)-B X_{-v}(h)\right) \\
& =\int_{\mathbb{R}}[1-\exp \{\mathrm{i} \lambda h\}]\left(\lambda^{2}+\alpha^{2}\right)^{-v} \mathrm{~d} \lambda .
\end{aligned}
$$

The change of variable $\lambda=\tilde{\lambda} /|h|$ in (2.8) leads to the following expression:

$$
\int_{\mathbb{R}}[1-\exp \{\mathrm{i}|\tilde{\lambda}| \cos \theta\}]\left(\tilde{\lambda}^{2}+(|h| \alpha)^{2}\right)^{-v}|h|^{2 v-1} \mathrm{~d} \tilde{\lambda}
$$

where $\theta$ represents the angle between $\lambda$ and $h$. From (2.9),

$$
\lim _{|h| \longrightarrow 0} \frac{\left[\mathrm{E}\left|\mathcal{X}_{-v}(s+h)-\mathcal{X}_{-v}(s)\right|^{2}\right]^{1 / 2}}{|h|^{v-1 / 2}}=K,
$$

where $K$ is a positive constant. Thus, for $|h| \in(0,1)$,

$$
\left[\mathrm{E}\left|\mathcal{X}_{-v}(s+h)-\mathcal{X}_{-v}(s)\right|^{2}\right]^{1 / 2} \leq C|h|^{\nu-1 / 2},
$$

which means that $\mathcal{X}_{-v}$ is mean-square Hölder continuous of order $v-\frac{1}{2}$.

From (2.10), $\mathcal{X}_{-v}$ is a $\left(v-\frac{1}{2}\right)$-index random field (see Adler (1981)) and, in the Gaussian case, Theorem 3.3 of Adler (1981, p. 57) implies that the sample paths of $\mathcal{X}_{v}$ have modulus of continuity $\Lambda(\delta)$ of order $\delta^{\nu-1 / 2}(|\ln \delta|)^{-1}$. Relation (2.7) also follows from Adler (1981, p. 204).

Next, we formulate the above results for model (2.1) in the $d$-dimensional case, i.e.

$$
(D+\alpha)^{v} X(x) \stackrel{\text { m.s. }}{=} \varepsilon(x), \quad x \in \mathbb{R}^{d},
$$

where

$$
D=\left(\frac{\partial}{\partial x_{1}} \cdots \frac{\partial}{\partial x_{d}}\right)
$$

and $\varepsilon$ is a generalized white noise on $L^{2}\left(\mathbb{R}^{d}\right)$; that is, $\varepsilon$ satisfies

$$
\mathrm{E}[\varepsilon(f) \varepsilon(g)]=\sigma^{2}\langle f, g\rangle_{L^{2}\left(\mathbb{R}^{d}\right)} .
$$

Specifically, the operator $(D+\alpha)^{v}$ can be expressed, in terms of the self-adjoint operator $\mathcal{L}=\left(\mathcal{L}_{x_{1}}, \ldots, \mathcal{L}_{x_{d}}\right)=\left(-\mathrm{i} \partial / \partial x_{1}, \ldots,-\mathrm{i} \partial / \partial x_{d}\right)$ on $L^{2}\left(\mathbb{R}^{d}\right)$, as

$$
(D+\alpha)^{v}=\left(\mathrm{i} \mathcal{L}_{x_{1}} \cdots \mathrm{i} \mathcal{L}_{x_{d}}+\alpha\right)^{\nu} .
$$

Then the covariance operator $R_{X_{-v}}$ admits the factorization

$$
R_{X_{-v}}=\left(\mathrm{i} \mathcal{L}_{x_{1}} \cdots \mathrm{i} \mathcal{L}_{x_{d}}+\alpha\right)^{-v}\left(-\mathrm{i} \mathcal{L}_{x_{1}} \cdots-\mathrm{i} \mathcal{L}_{x_{d}}+\alpha\right)^{-v}
$$


Both in the stationary and nonstationary cases, the class of fractional covariance models introduced in Ramm (1990) and Angulo et al. (2000a) can be studied in the generalized framework we consider. Note that the spectral density of the form

$$
f\left(\lambda_{1}, \lambda_{2}\right)=\frac{1}{\left(\lambda_{1}^{2}+\lambda_{2}^{2}-\alpha^{2}\right)^{3 / 2}}=\frac{1}{\left(|\lambda|^{2}-\alpha^{2}\right)^{3 / 2}}
$$

was introduced in the pioneering work of Whittle (1954).

From the spectral representation theorem (see, e.g. Dautray and Lions (1985b)), and from the embedding theorem between fractional Besov spaces (see, e.g. Triebel (1978)), the inverse Fourier transform of the spectral density (2.11) defines the kernel of the integral operator

$$
\left[(-\Delta)-\alpha^{2}\right]^{-3 / 2}
$$

where $\Delta$ denotes the Laplacian on $\mathbb{R}^{2}$. In a similar way to the proofs of the above results, it can be proved that the unique mean-square continuous solution to the equation

$$
\left[(-\Delta)-\alpha^{2}\right]^{3 / 4} X(x) \stackrel{\text { m.s. }}{=} \varepsilon(x), \quad x \in \mathbb{R}^{d},
$$

has the spectral density

$$
f\left(\lambda_{1}, \ldots, \lambda_{d}\right)=\frac{1}{\left(\sum_{i=1}^{d} \lambda_{i}^{2}-\alpha^{2}\right)^{3 / 2}}=\frac{1}{\left(|\lambda|^{2}-\alpha^{2}\right)^{3 / 2}},
$$

where now $\Delta$ denotes the Laplacian on $\mathbb{R}^{d}$. Model (2.11) corresponds to the case $d=2$. Similar results can be formulated for the more general model

$$
\left[(-\Delta)-\alpha^{2}\right]^{v / 2} X_{-v / 2}(x) \stackrel{\text { m.s. }}{=} \varepsilon(x), \quad x \in \mathbb{R}^{d}, v>0,
$$

with formal second-order solution of the form

$$
\int_{\mathbb{R}^{d}} \bar{g}(z) \mathcal{X}_{-v / 2}(z) \mathrm{d} z=\int_{\mathbb{R}^{d}} \bar{g}(z) \int_{\mathbb{R}^{d}} l(z, y) \mathrm{d} \varepsilon(y) \mathrm{d} z
$$

for $g \in L^{2}\left(\mathbb{R}^{d}\right)$, where

$$
l(z, y)=\int_{\mathbb{R}^{d}} \exp (\mathrm{i}\langle z-y, \lambda\rangle) \frac{1}{\left(|\lambda|^{2}-\alpha^{2}\right)^{v / 2}} \mathrm{~d} \lambda .
$$

The above mean-square solution is defined in the weak sense for $v \leq \frac{1}{2} d$ and in the strong sense for $v>\frac{1}{2} d$. Note that Whittle's (1954) model corresponds to the case $d=2$ and $v=\frac{3}{2}$, and Gay and Heyde's (1990) model corresponds to the case $d \geq 2$ and $v>0$. However, the spectral density (2.3) admits an alternative factorization (to (2.6)) of the associated covariance operator

$$
R_{\alpha, v}(g)(h)=\int_{\mathbb{R}} \int_{\mathbb{R}} h(t)\left[\int_{\mathbb{R}} \exp (\mathrm{i} \lambda(t-s)) f_{\alpha, v}(\lambda) \mathrm{d} \lambda\right] g(s) \mathrm{d} s \mathrm{~d} t \quad \text { for all } g, h \in L^{2}(\mathbb{R}),
$$

as follows:

$$
R_{\alpha, \nu}=\left((-\Delta)+\alpha^{2} I\right)^{-v / 2}\left((-\Delta)+\alpha^{2} I\right)^{-v / 2}
$$


This is written in terms of the self-adjoint integral operator $\left(\alpha^{2}-\Delta\right)^{-v / 2}$, which, in the case $\alpha=1$, coincides with the Bessel potential $I_{v}=(I-\Delta)^{-v / 2}$ of order $v$ with the kernel (see Stein (1970, pp. 131-132))

$$
I_{\nu}(z)=\frac{1}{(4 \pi)^{\nu / 2}} \frac{1}{\Gamma\left(\frac{1}{2} \nu\right)} \int_{0}^{\infty} \mathrm{e}^{-\pi|z|^{2} / \delta} \mathrm{e}^{-\delta / 4 \pi} \delta^{(-1+v) / 2} \frac{\mathrm{d} \delta}{\delta},
$$

in terms of the modified Bessel function of the third kind, of order $\frac{1}{2}(1-v)$ (see, e.g. Donoghue (1969)). The covariance factorization (2.12) corresponds to the spectral factorization

$$
f_{\alpha, v}(\lambda)=\left(\frac{1}{\left(|\lambda|^{2}+\alpha^{2}\right)^{v / 2}}\right)\left(\frac{1}{\left(|\lambda|^{2}+\alpha^{2}\right)^{v / 2}}\right) .
$$

In this case, the GRF $X_{-v}$ in Theorem 2.1 is defined as

$$
X_{-v}(h)=\varepsilon\left(\left(\alpha^{2} I-\Delta\right)^{-v / 2} h\right) \quad \text { for all } h \in L^{2}(\mathbb{R}),
$$

and the random field $X_{-v}$ is then given by

$$
\mathcal{X}_{-v}(t) \stackrel{\text { m.s. }}{=} \int_{\mathbb{R}} l(t, s) \mathrm{d} \varepsilon(s),
$$

where

$$
l(t, s)=(2 \pi)^{-1} \int_{\mathbb{R}} \exp (\mathrm{i} \lambda(t-s)) \frac{1}{\left(|\lambda|^{2}+\alpha^{2}\right)^{v / 2}} \mathrm{~d} \lambda .
$$

Note that (2.14) can be rewritten as

$$
\mathcal{X}_{-v}(t) \stackrel{\text { m.s. }}{=} \int_{\mathbb{R}} \exp (\mathrm{i} \lambda t) \frac{1}{\left(|\lambda|^{2}+\alpha^{2}\right)^{v / 2}} \mathrm{~d} \hat{\varepsilon}(\lambda),
$$

where $\hat{\varepsilon}$ represents generalized white noise.

From the above covariance factorization, the extension to $\mathbb{R}^{d}$ of the stochastic Laplace or stochastic Helmholtz equation of Gay and Heyde (1990) takes the form

$$
\left(\alpha^{2} I-\Delta\right)^{v / 2} X(x)=\varepsilon(x), \quad x \in \mathbb{R}^{d}, d \geq 2, v>0,
$$

where the operator $\left((-\Delta)+\alpha^{2}\right)^{v / 2}$ is defined by

$$
\left((-\Delta)+\alpha^{2}\right)^{\nu / 2}=(-1)^{\nu / 2} \sum_{j=0}^{\infty}\left(\begin{array}{c}
\frac{1}{2} v \\
j
\end{array}\right)(-\Delta)^{j} \alpha^{2(v / 2-j)} .
$$

The homogeneous isotropic solution $\mathcal{X}_{-v}$ to this equation has the spectral density

$$
f(\lambda)=\frac{\sigma^{2}}{(2 \pi)^{d}} \frac{1}{\left(|\lambda|^{2}+\alpha^{2}\right)^{v}}, \quad \lambda \in \mathbb{R}^{d},
$$

where $\mathcal{X}_{-v}$ is defined as in (2.14) with $l$ as in (2.15). Note that the kernel (2.13) is well defined in dimension $d$ (by replacing $\delta^{(-1+v) / 2}$ by $\left.\delta^{(-d+v) / 2}\right)$. 
Finally, the isotropic covariance function (see Yadrenko (1983))

$$
B(r)=(2 \pi)^{d} \int_{0}^{\infty}(r u)^{(2-d) / 2} J_{(d-2) / 2}(r u) u^{d-1} \frac{\sigma^{2}}{(2 \pi)^{d}} \frac{1}{\left(u^{2}+\alpha^{2}\right)^{v}} \mathrm{~d} u, \quad r>0, v>\frac{1}{2} d,
$$

belongs to the Matérn class (see Stein (1999, pp. 49-51)) and takes the form

$$
B(r)=\frac{\pi^{d / 2}}{\alpha^{2 v-d} 2^{\nu-d / 2-1} \Gamma(\nu)} \frac{\sigma^{2}}{(2 \pi)^{d}} K_{\nu-d / 2}(r \alpha)(r \alpha)^{\nu-d / 2}, \quad r>0, \alpha>0, v>\frac{1}{2} d,
$$

where again $K_{\lambda}$ is the Macdonald function with index $\lambda \in R$, and

$$
J_{\mu}(z)=\sum_{m=0}^{\infty} \frac{(-1)^{m}\left(\frac{1}{2} z\right)^{\mu+2 m}}{m ! \Gamma(\mu+m+1)}, \quad z>0,
$$

is the Bessel function of the first kind, of order $\mu$.

\section{Stochastic fractional evolution equations}

Let $X(t, x), t>0, x \in \mathbb{R}$, be a solution of the fully fledged stochastic partial differential equation (see Theorem 3.1 and Remark 3.1, below)

$$
\left[\frac{\partial}{\partial t}+\gamma-c^{2} \frac{\partial^{2}}{\partial x^{2}}\right]^{v} X(t, x)=\varepsilon(t, x), \quad t>0, c \geq 0, v>0, \gamma>0, x \in \mathbb{R}, c \in \mathbb{R},
$$

where $\varepsilon=\{\varepsilon(t, x), t>0, x \in \mathbb{R}\}$ is a white noise random field both in time and in space, i.e. $\varepsilon$ is a (generalized) zero-mean random field with covariance function

$$
\mathrm{E}[\varepsilon(f) \varepsilon(g)]=\sigma^{2}\langle f, g\rangle_{L^{2}\left(\mathbb{R}_{+} \times \mathbb{R}\right)}, \quad \sigma^{2}>0,
$$

for $f, g \in L^{2}\left(\mathbb{R}_{+} \times \mathbb{R}\right)$, the space of square-integrable functions on $\mathbb{R}_{+} \times \mathbb{R}$.

Heuristically, the second-order density of the m.s. solution to (3.1), that is stationary both in time and space, takes the form

$$
f_{\gamma, v}(\mu, \lambda)=\frac{\sigma^{2}}{(2 \pi)^{2}} \frac{1}{\left(\mu^{2}+\left(\gamma+c^{2} \lambda^{2}\right)^{2}\right)^{v}}, \quad \mu, \lambda \in \mathbb{R}, \gamma>0, \nu>1,
$$

where $\mu$ and $\lambda$ are, respectively, conjugates to $t$ and $x$.

An intuitively appealing approach to the spectral density (3.2) can be obtained by manipulation of divergent series, as follows. We expand the fractional heat operator as

$$
H_{v}=\left[\frac{\partial}{\partial t}+\gamma-c^{2} \frac{\partial^{2}}{\partial x^{2}}\right]^{v}=\left[\left(S+c D_{x}\right)\left(S-c D_{x}\right)\right]^{v},
$$

where, here and below,

$$
D_{x}=\frac{\partial}{\partial x}, \quad D_{t}=\frac{\partial}{\partial t}, \quad S=\left(D_{t}+\gamma\right)^{1 / 2}=\sum_{j=0}^{\infty}\left(\begin{array}{c}
\frac{1}{2} \\
j
\end{array}\right) D_{t}^{j} \gamma^{1 / 2-j}
$$


Next, we expand the fractional operators $\left(S+c D_{x}\right)^{v}$ and $\left(S-c D_{x}\right)^{v}$ in (3.1) and (3.3) as follows:

$$
\begin{aligned}
& \left(S+c D_{x}\right)^{\nu}=\sum_{k=0}^{\infty}\left(\begin{array}{l}
\nu \\
k
\end{array}\right) c^{k} D_{x}^{k} S^{\nu-k}=\sum_{k=0}^{\infty}\left(\begin{array}{l}
\nu \\
k
\end{array}\right) c^{k} D_{x}^{k}\left(D_{t}+\gamma\right)^{(\nu-k) / 2} \\
& =\sum_{k=0}^{\infty} \sum_{j=0}^{\infty}\left(\begin{array}{l}
v \\
k
\end{array}\right)\left(\begin{array}{c}
\frac{1}{2}(v-k) \\
j
\end{array}\right) c^{k} D_{x}^{k} D_{t}^{j} \gamma^{(v-k) / 2-j} \\
& \left(S-c D_{x}\right)^{v}=(-1)^{v} \sum_{k^{\prime}=0}^{\infty} \sum_{j^{\prime}=0}^{\infty}(-1)^{k^{\prime}}\left(\begin{array}{c}
v \\
k^{\prime}
\end{array}\right)\left(\begin{array}{c}
\frac{1}{2}\left(v-k^{\prime}\right) \\
j^{\prime}
\end{array}\right) c^{k^{\prime}} D_{x}^{k^{\prime}} D_{t}^{j^{\prime}} \gamma^{\left(v-k^{\prime}\right) / 2-j^{\prime}} .
\end{aligned}
$$

Thus, we obtain from (3.3)-(3.5) the following (divergent) series for the fractional heat operator:

$$
\begin{aligned}
H_{v}= & (-1)^{v} \sum_{k=0}^{\infty} \sum_{j=0}^{\infty} \sum_{k^{\prime}=0}^{\infty} \sum_{j^{\prime}=0}^{\infty}(-1)^{k^{\prime}}\left(\begin{array}{c}
v \\
k
\end{array}\right)\left(\begin{array}{c}
\frac{1}{2}(v-k) \\
j
\end{array}\right)\left(\begin{array}{c}
v \\
k^{\prime}
\end{array}\right)\left(\begin{array}{c}
\frac{1}{2}\left(v-k^{\prime}\right) \\
j^{\prime}
\end{array}\right) \\
& \times c^{k+k^{\prime}} D_{x}^{k+k^{\prime}} D_{t}^{j+j^{\prime}} \gamma^{\nu-\left(k+k^{\prime}\right) / 2-\left(j+j^{\prime}\right) / 2}
\end{aligned}
$$

This implies the form (3.2) for the spectral density of the solution of the equation

$$
H_{v} X(t, x)=\varepsilon(t, x) .
$$

Note that, for $v=1$, the spectral density (3.2) was obtained by Whittle (1963) by using the above approach. Moreover, Christakos (2000, p. 225) suggested, in a geostatistical context, a set of covariances in $(0, \infty) \times \mathbb{R}^{d}$ having the spatiotemporal spectral density

$$
f(\mu, \lambda)=\frac{\sigma_{1}^{2}}{\left[c_{1}^{2} \mu^{2}+\left(a^{2}+|\lambda|^{2}\right)^{2 p}\right]}, \quad \mu \in \mathbb{R}, \lambda \in \mathbb{R}^{d}, n \geq 1 .
$$

In the case $p=1, d=1$, and $c_{1}^{2}=1$, this formula reduces to spectral density (3.2) with $v=1, \sigma_{1}^{2}=\sigma^{2} /(2 \pi)^{2}, c_{1}^{2}=1$, and $a^{2}=\gamma$.

For $\gamma>0$ and $v>1$, the random field with spectral density (3.2) exhibits a short-range dependence both in time and in space simultaneously, that is,

$$
\lim _{\max \{\lambda, \mu\} \rightarrow 0} f_{\gamma, v}(\mu, \lambda)=\frac{\sigma^{2}}{(2 \pi)^{2}} \frac{1}{\gamma^{2 v}}>0 ;
$$

and in time and space separately, that is, for a fixed $\lambda \in \mathbb{R}$,

$$
\lim _{\mu \rightarrow 0} f_{\gamma, v}(\mu, \lambda)=\frac{\sigma^{2}}{(2 \pi)^{2}} \frac{1}{\left(\gamma+c^{2} \lambda^{2}\right)^{2 v}}>0
$$

and, for a fixed $\mu>0$,

$$
\lim _{\lambda \rightarrow 0} f_{\gamma, v}(\mu, \lambda)=\frac{\sigma^{2}}{(2 \pi)^{2}} \frac{1}{\left(\mu^{2}+\gamma^{2}\right)^{v}}>0 .
$$


However, the random field with spectral density (3.2) displays the following fractal behaviour at infinity:

$$
f_{\gamma, v}(\mu, \lambda)=O\left((\min \{\mu, \lambda\})^{-2 v}\right) \quad \text { as } \min \{\mu, \lambda\} \rightarrow \infty .
$$

This holds in time and space separately; that is, for a fixed $\lambda \in \mathbb{R}$,

$$
f_{\gamma, v}(\mu, \lambda)=O\left(\mu^{-2 v}\right), \quad \mu \rightarrow \infty,
$$

and, for a fixed $\mu \in \mathbb{R}$,

$$
f_{\gamma, v}(\mu, \lambda)=O\left(\lambda^{-4 v}\right), \quad \lambda \rightarrow \infty .
$$

This random field also displays long-range dependence both in time and in space in the particular case $\gamma=0$, since

$$
\lim _{\max \{\lambda, \mu\} \rightarrow 0} f_{0, v}(\mu, \lambda)=\infty .
$$

However, for $\gamma=0$ and $0<v<1$, (3.2) does not represent the spectral density of a secondorder stationary random field, but rather of a self-similar random field with homogeneous increments. In particular, the second-order moments of the random field $X\left(t a, x a^{1 / 2}\right)$ coincide with those of $a^{v-3 / 4} X(t, x)$ for every $a>0$ and $v \in\left(\frac{3}{4}, \frac{7}{4}\right)$.

If $\gamma=0$ and $v \in\left(0, \frac{1}{4}\right)$, the random field

$$
v(t, x)=\int_{t}^{t+1} \int_{x}^{x+1} X(s, y) \mathrm{d} s \mathrm{~d} y, \quad t>0, x \in \mathbb{R},
$$

has the spectral density

$$
h(\mu, \lambda)=\frac{\sigma^{2}}{(2 \pi)^{2}} \frac{\sin ^{2}\left(\frac{1}{2} \mu\right)}{\left(\frac{1}{2} \mu\right)^{2}} \frac{\sin ^{2}\left(\frac{1}{2} \lambda\right)}{\left(\frac{1}{2} \lambda\right)^{2}} \frac{1}{\left(\mu^{2}+c^{4} \lambda^{4}\right)^{v}}, \quad \mu, \lambda \in \mathbb{R} .
$$

Therefore, it is stationary both in time and in space and displays long-range dependence both in time and in space; that is,

$$
h(\mu, \lambda) \sim \frac{\sigma^{2}}{(2 \pi)^{2}} \frac{1}{\left(\mu^{2}+c^{4} \lambda^{4}\right)^{v}} \quad \text { as } \max \{\lambda, \mu\} \rightarrow 0,
$$

and

$$
h(\mu, \lambda)=O\left(\frac{1}{\mu^{2} \lambda^{2}\left(\mu^{2}+c^{4} \lambda^{4}\right)^{v}}\right) \quad \text { as } \min \{\lambda, \mu\} \rightarrow \infty .
$$

In order to make rigorous the heuristic derivation of (3.2) from (3.1), we will again use the theory of GRFs on fractional Sobolev spaces.

The operator $H_{v}$ is a function of the elliptic self-adjoint differential operator $\left(\mathcal{L}_{t}, \mathcal{L}_{x}\right)=$ $(-\mathrm{i} \partial / \partial t,-\mathrm{i} \partial / \partial x)$, densely defined on the separable Hilbert space $\left(L^{2}\left(\mathbb{R}_{+} \times \mathbb{R}\right), \mathbb{C}\right)$. Specifically,

$$
H_{\nu}=\left(\mathrm{i} \mathcal{L}_{t}+\gamma+c^{2} \mathcal{L}_{x}^{2}\right)^{v} .
$$

Hence, for each $\varphi \in \mathscr{D}\left(H_{v}\right), H_{v}$ admits the spectral representation

$$
\begin{aligned}
H_{\nu}(\varphi)(g) & =\int_{\Lambda_{t} \times \Lambda_{x}}\left[\mathrm{i} \mu+\gamma+c^{2} \lambda^{2}\right]^{v} \mathrm{~d}\left(E_{(\mu, \lambda)}(\varphi), g\right) \\
& =\left(\frac{\partial}{\partial t}+\gamma-c^{2} \frac{\partial^{2}}{\partial x^{2}}\right)^{v}(\varphi)(g) \quad \text { for all } g \in L^{2}\left(\mathbb{R}_{+} \times \mathbb{R}\right),
\end{aligned}
$$


where $\Lambda_{t} \times \Lambda_{x}$ stands for the continuous spectrum of the operator $\left(\mathcal{L}_{t}, \mathcal{L}_{x}\right)$ and $\left\{E_{(\mu, \lambda)}\right.$ : $\left.(\mu, \lambda) \in \Lambda_{t} \times \Lambda_{x}\right\}$ denotes its spectral family.

The formal adjoint $H_{v}^{\dagger}$ of $H_{v}$ is then represented, for each $\varphi \in \mathscr{D}\left(H_{v}^{\dagger}\right)=\mathscr{D}\left(H_{v}\right)$, as

$$
\begin{aligned}
H_{v}^{\dagger}(\varphi)(g) & =\int_{\Lambda_{t} \times \Lambda_{x}}\left[-\mathrm{i} \mu+\gamma+c^{2} \lambda^{2}\right]^{v} \mathrm{~d}\left(E_{(\mu, \lambda)}(\varphi), g\right) \\
& =\left(-\mathrm{i} \mathcal{L}_{t}+\gamma+c^{2} \mathcal{L}_{x}^{2}\right)^{v}(\varphi)(g) \\
& =\left(-\frac{\partial}{\partial t}+\gamma-c^{2} \frac{\partial^{2}}{\partial x^{2}}\right)^{v}(\varphi)(g) \quad \text { for all } g \in L^{2}\left(\mathbb{R}_{+} \times \mathbb{R}\right),
\end{aligned}
$$

where we have used the fact that $-\mathrm{i} \mathcal{L}_{t}=-\partial / \partial t$ is the formal adjoint of $\mathrm{i} \mathcal{L}_{t}=\partial / \partial t$.

In terms of the spectral representations of $H_{v}$ and $H_{v}^{\dagger}$, their common domain $\mathscr{D}\left(H_{v}\right)=$ $\mathscr{D}\left(H_{v}^{\dagger}\right)$ is defined as

$$
\mathscr{D}\left(H_{\nu}\right)=\left\{f \in L^{2}\left(\mathbb{R}_{+} \times \mathbb{R}\right): \int_{\Lambda_{t} \times \Lambda_{x}}\left(\mu^{2}+\left(\gamma+c^{2} \lambda^{2}\right)^{2}\right)^{v} \mathrm{~d}\left(E_{(\mu, \lambda)}(f), f\right)<\infty\right\} .
$$

Note that, for each $\varphi \in \mathscr{D}\left(H_{v}^{\dagger}\right)=\mathscr{D}\left(H_{v}\right)$,

$$
H_{\nu} H_{\nu}^{\dagger}(\varphi)(\psi)=\int_{\Lambda_{t} \times \Lambda_{x}}\left(\mu^{2}+\left(\gamma+c^{2} \lambda^{2}\right)^{2}\right)^{v} \mathrm{~d}\left(E_{(\mu, \lambda)}(\varphi), \psi\right) \quad \text { for all } \psi \in \mathscr{D}\left(H_{v}^{\dagger}\right) .
$$

The inverse operator $H_{v}^{-1}$ of $H_{v}$ admits a similar spectral representation; that is, for each $f \in \mathscr{D}\left(H_{v}^{-1}\right)$,

$$
\begin{aligned}
H_{v}^{-1}(f)(g) & =\int_{\Lambda_{t} \times \Lambda_{x}}\left(\mathrm{i} \mu+\gamma+c^{2} \lambda^{2}\right)^{-v} \mathrm{~d}\left(E_{(\mu, \lambda)}(f), g\right) \\
& =\left(\mathrm{i} \mathcal{L}_{t}+\gamma+c^{2} \mathcal{L}_{x}^{2}\right)^{-v}(f)(g) \quad \text { for all } g \in L^{2}\left(\mathbb{R}_{+} \times \mathbb{R}\right) .
\end{aligned}
$$

As the operator $\left(\mathcal{L}_{t}, \mathcal{L}_{x}\right)$ satisfies conditions given in Ramm (1990, pp. 145-148), the projection operators $E_{(\mu, \lambda)},(\mu, \lambda) \in \Lambda_{t} \times \Lambda_{x}$, defining its spectral family, admit an integral representation in terms of a kernel given by

$$
E_{(\mu, \lambda)}(t, s ; x, y ; \mu, \lambda)=\int_{-\infty}^{\lambda} \int_{-\infty}^{\mu} \Phi(t, s ; x, y ; \xi, \omega) \mathrm{d} \rho(\xi, \omega),
$$

where $\Phi(t, s ; x, y ; \xi, \omega)=(2 \pi)^{-2} \exp (\mathrm{i}\langle(t-s, x-y),(\xi, \omega)\rangle)$ represents the spectral kernel and $\mathrm{d} \rho(\xi, \omega)=\mathrm{d} \xi \mathrm{d} \omega$ is the spectral measure of the operator $\left(\mathscr{L}_{t}, \mathcal{L}_{x}\right)$. The above spectral representations of the operators $H_{v}, H_{v}^{\dagger}$, and $H_{v} H_{v}^{\dagger}$ can be expressed in terms of $\Phi$ and $\rho$ in a similar way as in the previous section (for the operator $\left.(D+\alpha)^{v}\right)$.

Theorem 3.1. Let $X_{-v}$ be a GRF defined as

$$
\begin{gathered}
X_{-v}(h) \stackrel{\text { m.s. }}{=} \int_{\mathbb{R}_{+} \times \mathbb{R}} \int_{\mathbb{R}_{+} \times \mathbb{R}} \bar{h}(t, x) l(t, x ; s, y) \mathrm{d} \varepsilon(s, y) \mathrm{d} x \mathrm{~d} t \\
\stackrel{\text { m.s. }}{=} \int_{\mathbb{R}_{+} \times \mathbb{R}} \bar{h}(t, x) \mathcal{X}_{-v}(t, x) \mathrm{d} x \mathrm{~d} t
\end{gathered}
$$


for all $h \in L^{2}\left(\mathbb{R}_{+} \times \mathbb{R}\right)$, where $\varepsilon$ is generalized white noise, as in (3.1), and

$$
l(t, x ; s, y)=(2 \pi)^{-2} \int_{\Lambda_{t} \times \Lambda_{x}} \exp (\mathrm{i}\langle(t-s, x-y),(\mu, \lambda)\rangle)\left(\mathrm{i} \mu+\gamma+c^{2} \lambda^{2}\right)^{-v} \mathrm{~d} \lambda \mathrm{d} \mu .
$$

Then for $v \leq \frac{2}{3}, X_{-v}$ defines - in the weak sense - a mean-square solution $X_{-v}$ to (3.1) and, for $v>\frac{2}{3}, X_{-v}$ defines - in the strong sense - the unique mean-square continuous solution $X_{-v}$ to $(3.1)$.

The GRF $X_{-v}$ has RKHS

$$
\mathscr{H}\left(X_{-\nu}\right)=\left\{\phi \in L^{2}\left(\mathbb{R}_{+} \times \mathbb{R}\right): \int_{\Lambda_{t} \times \Lambda_{x}}\left(\mu^{2}+\left(\gamma+c^{2} \lambda^{2}\right)^{2}\right)^{v}|\hat{\phi}(\mu, \lambda)|^{2} \mathrm{~d} \lambda \mathrm{d} \mu<\infty\right\},
$$

with the inner product

$$
\langle\phi, \varphi\rangle_{\mathscr{H}\left(X_{-v}\right)}=\int_{\Lambda_{t} \times \Lambda_{x}}\left(\mu^{2}+\left(\gamma+c^{2} \lambda^{2}\right)^{2}\right)^{v} \hat{\phi}(\mu, \lambda) \overline{\hat{\varphi}}(\mu, \lambda) \mathrm{d} \lambda \mathrm{d} \mu,
$$

where a 'hat' (caret) over a function denotes the projection of the function in terms of the spectral kernel of the linear operator considered.

Remark 3.1. The mean-square fractional regularity order of the GRF $X_{-v}$ defined in (3.6), that is, the weak-sense regularity order of the functions of its RKHS, is $\frac{3}{2} v=\frac{1}{2}(v+2 v)$, with fractional regularity order $v$ in time and fractional regularity order $2 v$ in space $(v>0)$. Its minimum mean-square fractional singularity order, that is, the weak-sense regularity order of the test functions defining its domain, is then $-\frac{3}{2} \nu$. Although, in the formulation of Theorem 3.1, we consider the separable Hilbert space $L^{2}\left(\mathbb{R}_{+} \times \mathbb{R}\right), X_{-v}$ can be defined on a larger function space, according to its regularity order.

Proof of Theorem 3.1. Random field $X_{-v}$ defined in (3.6) satisfies the mean-square identity

$$
\begin{aligned}
X_{-v}(h) & \stackrel{\text { m.s }}{=} \int_{\mathbb{R}_{+} \times \mathbb{R}}\left[\overline{\int_{\mathbb{R}_{+} \times \mathbb{R}} \tilde{l}(s, y ; t, x) h(t, x) \mathrm{d} x \mathrm{~d} t}\right] \mathrm{d} \varepsilon(s, y) \\
& \stackrel{\text { m.s }}{=} \varepsilon\left[\left(-\frac{\partial}{\partial t}+\gamma-c^{2} \frac{\partial^{2}}{\partial x^{2}}\right)^{-v} h\right] \text { for all } h \in L^{2}\left(\mathbb{R}_{+} \times \mathbb{R}\right),
\end{aligned}
$$

where

$$
\widetilde{l}(t, x ; s, y)=\frac{1}{(2 \pi)^{2}} \int_{\Lambda_{t} \times \Lambda_{x}} \exp (\mathrm{i}\langle(t-s, x-y),(\mu, \lambda)\rangle)\left(-\mathrm{i} \mu+\gamma+c^{2} \lambda^{2}\right)^{-v} \mathrm{~d} \lambda \mathrm{d} \mu
$$

is the kernel of the integral operator $\left(-\partial / \partial t+\gamma-c^{2} \partial^{2} / \partial x^{2}\right)^{-v}$.

Therefore,

$$
\begin{aligned}
X_{-v}\left(H_{v}^{\dagger} \varphi\right) & \stackrel{\text { m.s }}{=} \varepsilon\left[\left(-\frac{\partial}{\partial t}+\gamma-c^{2} \frac{\partial^{2}}{\partial x^{2}}\right)^{-v}\left(-\frac{\partial}{\partial t}+\gamma-c^{2} \frac{\partial^{2}}{\partial x^{2}}\right)^{v} \varphi\right] \\
& \stackrel{\text { m.s }}{=} \varepsilon(\varphi) \quad \text { for all } \varphi \in \mathcal{D}\left(H_{v}^{\dagger}\right) .
\end{aligned}
$$

That is, $X_{-v}$ satisfies, in the mean-square sense, the generalized equation on $\mathscr{D}\left(H_{v}^{\dagger}\right)$ associated with (3.1). 
From embedding theorems between fractional Besov spaces, for $v>\frac{2}{3}$,

$$
\chi_{-v}(t, x) \stackrel{\text { m.s }}{=} \int_{\mathbb{R}_{+} \times \mathbb{R}} l(t, x ; s, y) \mathrm{d} \varepsilon(s, y)
$$

defines the unique mean-square, continuous, ordinary solution to (3.1), with $l$ defined as in (3.7). This implies that the spectral density of $\mathcal{X}_{-v}$ is given by (3.2).

The definition of the reproducing kernel Hilbert space $\mathscr{H}\left(X_{-v}\right)$ of $X_{-v}$ is as in the theory of GRFs on fractional Sobolev spaces developed in Ruiz-Medina et al. (2001), (2004), and is motivated by the following definition of the dual random field $\widetilde{X}_{-v}$ of $X_{-v}$ :

$$
\tilde{X}_{-v}(\psi)=\varepsilon\left[\left(\frac{\partial}{\partial t}+\gamma-c^{2} \frac{\partial^{2}}{\partial x^{2}}\right)^{v} \psi\right] \text { for all } \psi \in \mathcal{D}\left(H_{\nu}\right)
$$

Hence,

$$
\begin{aligned}
\langle\phi, \varphi\rangle_{\mathscr{H}\left(X_{-v}\right)} & =\left\langle\tilde{X}_{-v}(\phi), \tilde{X}_{-v}(\varphi)\right\rangle_{H\left(X_{-v}\right)=H\left(\tilde{X}_{-v}\right)} \\
& =\int_{\Lambda_{t} \times \Lambda_{x}}\left(\mathrm{i} \mu+\gamma+c^{2} \lambda^{2}\right)^{v} \hat{\phi}(\mu, \lambda) \overline{\left(\mathrm{i} \mu+\gamma+c^{2} \lambda^{2}\right)^{v} \hat{\varphi}(\mu, \lambda)} \mathrm{d} \lambda \mathrm{d} \mu \\
& =\int_{\Lambda_{t} \times \Lambda_{x}}\left(\mu^{2}+\left(\gamma+c^{2} \lambda^{2}\right)^{2}\right)^{v} \hat{\phi}(\mu, \lambda) \overline{\hat{\varphi}}(\mu, \lambda) \mathrm{d} \lambda \mathrm{d} \mu .
\end{aligned}
$$

The definition of $\tilde{X}_{-v}$ guarantees the bicontinuity of the covariance operator $R_{X_{-v}}$, and the closeness of such an operator. Thus, the RKHS of $X_{-v}$ is a (closed) Hilbert space with norm generated by the inner product (3.8), and is a dense subspace of $L^{2}\left(\mathbb{R}_{+} \times \mathbb{R}\right)$.

The extension of Theorem 3.1 to the $d$-dimensional case is straightforward using the relation

$$
H_{v}=\left(\mathrm{i} \mathcal{L}_{t}+\gamma+c^{2} \mathcal{L}_{x}^{2}\right)^{v},
$$

with $\mathcal{L}_{x}^{2}=-\Delta$ and $\Delta$ the Laplacian operator on $\mathbb{R}^{d}$ as before. Hence, $H_{v}$ admits the spectral representation

$$
H_{\nu}(\varphi)(g)=\int_{\Lambda_{t} \times \Lambda_{x}}\left(\mathrm{i} \mu+\gamma+c^{2}|\lambda|^{2}\right)^{v} \mathrm{~d}\left(E_{(\mu, \lambda)}(\varphi), g\right) \quad \text { for all } g \in L^{2}\left(\mathbb{R}_{+} \times \mathbb{R}^{d}\right)
$$

and for each $\varphi \in \mathscr{D}\left(H_{v}\right)$, where $\Lambda_{t} \times \Lambda_{x}$ stands for the continuous spectrum of the selfadjoint operator $\left(\mathcal{L}_{t}, \mathcal{L}_{x}\right)=\left(-\mathrm{i} \partial / \partial t,-\mathrm{i} \partial / \partial x_{1}, \ldots,-\mathrm{i} \partial / \partial x_{d}\right)$ on $L^{2}\left(\mathbb{R}_{+} \times \mathbb{R}^{d}\right)$, and $\left\{E_{(\mu, \lambda)}\right.$ : $\left.(\mu, \lambda) \in \Lambda_{t} \times \Lambda_{x}\right\}$ is its spectral family.

In a similar manner, one can view many useful fractional differential spatiotemporal models as fractional versions of the heat equation.

Let $\mathcal{X}_{-1,-p}$ be the mean-square solution to

$$
\left[c \frac{\partial}{\partial t}+\left(-\Delta+a^{2}\right)^{p}\right] \mathcal{X}_{-1,-p} \stackrel{\text { m.s. }}{=} \varepsilon,
$$

where $\Delta$ represents the Laplacian on $\mathbb{R}^{d}$ and $\varepsilon$ denotes spatiotemporal white noise with intensity $\sigma$. For $p>\left(d^{2}+2 d-1\right) / 4 d, \mathcal{X}_{-1,-p}$ is defined in the strong sense as

$$
\chi_{-1,-p}(t, x) \stackrel{\text { m.s. }}{=} \int_{T \times \mathbb{R}^{d}} l(t, x ; s, y) \mathrm{d} \varepsilon(s, y) \quad \text { for all }(t, x) \in L^{2}\left(T \times \mathbb{R}^{d}\right),
$$


where

$$
l(t, x ; s, y)=(2 \pi)^{-d-1} \int_{\Lambda_{t} \times \Lambda_{x}} \exp (\mathrm{i}\langle(t-s, x-y),(\mu, \lambda)\rangle) \frac{1}{\mathrm{i} c \mu+\left(|\lambda|^{2}+a^{2}\right)^{p}} \mathrm{~d} \lambda \mathrm{d} \mu
$$

with associated spectral density

$$
f_{1, p}(\mu, \lambda)=\frac{1}{c^{2} \mu^{2}+\left(|\lambda|^{2}+a^{2}\right)^{2 p}}
$$

(cf. Christakos (2000) and Jones and Zhang (1997)).

For $p \leq\left(d^{2}+2 d-1\right) / 4 d, X_{-1,-p}$ is defined in terms of a generalized spatiotemporal random field

$$
X_{-1,-p}(h)=\varepsilon\left(\left[-c \frac{\partial}{\partial t}+\left((-\Delta)+a^{2}\right)^{p}\right]^{-1} h\right) \text { for all } h \in L^{2}\left(T \times \mathbb{R}^{d}\right) .
$$

Note that the mean-square fractional regularity order of $X_{-1,-p}$ is $(1+2 p d) /(d+1)$, with 1 representing the regularity order in time and $2 p$ the regularity order in space.

Model (3.9) is included in a more general family

$$
\left(\frac{\partial}{\partial t}+L_{x}^{v}\right) X_{-1,-v} \stackrel{\text { m.s. }}{=} \varepsilon
$$

where $L_{x}^{v}$ is a spatial linear self-adjoint differential operator of order $v$ on $L^{2}\left(\mathbb{R}^{d}\right)$. In the case where $L_{x}^{v}=F_{v}(-\Delta)$, i.e. a function of $-\Delta$ (with $\Delta$ the Laplacian operator on $\mathbb{R}^{d}$ ) involving fractional powers of this operator, and $T$ is an unbounded interval (e.g. $T=\mathbb{R}_{+}$), a fractional version of the heat equation emerges with m.s. solution given by

$$
\chi_{-1,-v}(t, x) \stackrel{\text { m.s. }}{=} \int_{\mathbb{R}_{+} \times \mathbb{R}^{d}} l(t, x ; s, y) \mathrm{d} \varepsilon(s, y)
$$

for $(1+v d) /(d+1)>\frac{1}{2}(d+1)$, where

$$
l(t, x ; s, y)=(2 \pi)^{-d-1} \int_{\Lambda_{t} \times \Lambda_{x}} \exp (\mathrm{i}\langle(t-s, x-y),(\mu, \lambda)\rangle) \frac{1}{\mathrm{i} \mu+F_{\nu}\left(|\lambda|^{2}\right)} \mathrm{d} \lambda \mathrm{d} \mu .
$$

Its spectral density is then defined as

$$
f_{1, v}(\mu, \lambda)=\frac{1}{\mu^{2}+\left(F_{v}\left(|\lambda|^{2}\right)\right)^{2}} .
$$

In particular, model (3.9) corresponds to the case where $v=2 p$, and $F_{v}(-\Delta)=$ $\left((-\Delta)+a^{2}\right)^{p}$. If $F_{v}(-\Delta)=(I-\Delta)^{\alpha / 2}(-\Delta)^{\gamma / 2}$ (i.e. $\left.v=\alpha+\gamma\right)$, we obtain the fractional heat equation considered in Angulo et al. (2000b).

Now consider $Y(t, x), t>0, x \in \mathbb{R}^{d}$, a spatiotemporal random field defined as the output of the following fractional differential filter applied to a spatiotemporal random field $\mathcal{X}_{-\beta,-v}$ with $v+1=\sum_{i=1}^{d} v_{i}$ :

$$
\frac{\partial^{v+\beta+2}}{\partial x_{1}^{\nu_{1}} \partial x_{2}^{\nu_{2}} \cdots \partial x_{d}^{\nu_{d}} \partial t^{\beta+1}} \chi_{-\beta,-v}=Y(t, x), \quad \beta>0, v_{i}>0, i=1, \ldots, d .
$$


Here, $Y$ is assumed to be a zero-mean, spatially homogeneous, temporally stationary field. Thus, $Y$ belongs to the class of spatiotemporal random fields (called $\mathrm{S} / \mathrm{TRF}_{\nu / \beta}$ models) considered in Christakos (1991), (2000) and can be interpreted as a generalized spatiotemporal random field defined from $\mathcal{X}_{-\beta,-v}$ in terms of the test function family (see Christakos (2000, p. 255))

$$
\begin{aligned}
q_{t, x}(s, y)= & (2 \pi)^{-(d+1)} \int_{\Lambda_{t} \times \Lambda_{x}}\left(\mathrm{i} \lambda_{1}\right)^{\nu_{1}} \cdots\left(\mathrm{i} \lambda_{d}\right)^{v_{d}}(\mathrm{i} \mu)^{(\beta+1)} \\
& \times \exp (\mathrm{i}\langle(t-s, x-y),(\mu, \lambda)\rangle) \mathrm{d} \lambda \mathrm{d} \mu,
\end{aligned}
$$

where $(t, x) \in \mathbb{R}_{+} \times \mathbb{R}^{d}, \Lambda_{t} \times \Lambda_{x}$ is the continuous spectrum of the operator

$$
\left(\mathcal{L}_{x_{1}}, \ldots, \mathcal{L}_{x_{d}}, \mathcal{L}_{t}\right)=\left(-\mathrm{i} \frac{\partial}{\partial x_{1}}, \ldots,-\mathrm{i} \frac{\partial}{\partial x_{d}},-\mathrm{i} \frac{\partial}{\partial t}\right),
$$

and $(2 \pi)^{-(d+1)} \exp (\mathrm{i}\langle(t-s, x-y),(\mu, \lambda)\rangle)$ is its spectral kernel. Note that the operator

$$
\delta^{\nu+\beta+2}=\frac{\partial^{\nu+\beta+2}}{\partial x_{1}^{\nu_{1}} \partial x_{2}^{\nu_{2}} \cdots \partial x_{d}^{\nu_{d}} \partial t^{\beta+1}}
$$

can be defined via the operator $\left(\mathcal{L}_{x_{1}}, \ldots, \mathcal{L}_{x_{d}}, \mathcal{L}_{t}\right)$ in $(3.10)$ as

$$
\delta^{v+\beta+2}=\left(\mathcal{L}_{x_{1}}\right)^{\nu_{1}} \cdots\left(\mathcal{L}_{x_{d}}\right)^{\nu_{d}}\left(\mathcal{L}_{t}\right)^{\beta+1},
$$

with $\mathscr{D}\left(\delta^{\nu+\beta+2}\right)=H^{\nu+\beta+2}\left(\mathbb{R}_{+} \times \mathbb{R}^{d}\right)$ dense in $L^{2}\left(\mathbb{R}_{+} \times \mathbb{R}^{d}\right)$. The parametric family of $(\nu / \beta)$-random field models defined above provides a useful tool in heterogeneity analysis in modern spatiotemporal geostatistics. This parametric family allows us, by properly selecting the test functions $q$, to represent the degree of departure from homogeneity (parameter $v$ ) and from stationarity (parameter $\beta$ ) (see Christakos (2000)).

In the case where random field $Y$ is a generalized white noise on $L^{2}\left(\mathbb{R}_{+} \times \mathbb{R}^{d}\right)$, it can be proved similarly to Theorem 3.1 that $\mathcal{X}_{-\beta,-v}$ is well defined, in the mean-square sense, by

$$
\begin{aligned}
& \int_{\mathbb{R}_{+} \times \mathbb{R}^{d}} \bar{g}(t, x) \mathcal{X}_{-\beta,-v}(t, x) \mathrm{d} x \mathrm{~d} t \\
& \stackrel{\text { m.s. }}{=} \int_{\mathbb{R}_{+} \times \mathbb{R}^{d}} \bar{g}(t, x) \int_{\mathbb{R}_{+} \times \mathbb{R}^{d}} l(t, x ; s, y) \mathrm{d} \varepsilon(s, y) \mathrm{d} x \mathrm{~d} t
\end{aligned}
$$

for $g \in L^{2}\left(\mathbb{R}_{+} \times \mathbb{R}^{d}\right)$, where

$$
\begin{aligned}
l(t, x ; s, y)= & (2 \pi)^{-(d+1)} \int_{\Lambda_{t} \times \Lambda_{x}}\left(\mathrm{i} \lambda_{1}\right)^{-\nu_{1}} \cdots\left(\mathrm{i} \lambda_{d}\right)^{-v_{d}}(\mathrm{i} \mu)^{-(\beta+1)} \\
& \times \exp (\mathrm{i}\langle(t-s, x-y),(\mu, \lambda)\rangle) \mathrm{d} \lambda \mathrm{d} \mu .
\end{aligned}
$$

\section{Renormalization and homogenization}

Gaussian and non-Gaussian limiting distributions for the renormalized solutions of the heat equation fractional in space and/or in time, with random initial conditions, have been studied in Anh and Leonenko (2000), (2001), (2002). However, our equation (3.1) differs both in form and by rescaling (see Theorem 4.1, below) from the fractional heat equations of those references, and also those of Mainardi et al. (2001) (see also the references therein). 
The goal of this section is to state and prove a functional central limit theorem for the polynomials of the random field

$$
X(t, x ; \gamma)=\int_{\mathbb{R}^{2}} \mathrm{e}^{\mathrm{i} \mu t+\mathrm{i} \lambda x} f_{\gamma, v}^{1 / 2}(\mu, \lambda) Z(\mathrm{~d} \mu, \mathrm{d} \lambda), \quad t>0, \gamma>0, v>1, x \in \mathbb{R},
$$

where $f_{\gamma, v}$ is defined by (3.2) and $Z$ is a complex-valued white noise random measure, that is,

$$
\mathrm{E}[Z(A)]=0, \quad \mathrm{E}[Z(A) \overline{Z(B)}]=|A \cap B|, \quad A, B \in \mathscr{B},
$$

where $\mathscr{B}$ is the Borel $\sigma$-field of $\mathbb{R}^{2}$.

In view of the results of Section 3, the random field (4.1) can be considered as the m.s. solution of the fractional stochastic evolution equation (3.1).

If $Z(A)=Z_{G}(A)$ is a Gaussian random measure then, in distribution,

$$
Z_{G}(a \mathrm{~d} \mu, \sqrt{a} \mathrm{~d} \lambda) \stackrel{\mathrm{D}}{=} a^{3 / 4} Z_{G}(\mathrm{~d} \mu, \mathrm{d} \lambda), \quad a>0
$$

where ' $\stackrel{D}{=}$ denotes equality in distribution. This identity implies that the random field (4.1) with Gaussian random measure $Z=Z_{G}$ is self-similar, in the sense that, for any $\epsilon>0$,

$$
X\left(\frac{t}{\epsilon}, \frac{x}{\sqrt{\epsilon}} ; \gamma \epsilon\right) \stackrel{\mathrm{D}}{=} \epsilon^{3 / 4-v} X(t, x, \gamma), \quad t>0, v>1, \gamma>0, x \in \mathbb{R}
$$

Considering a linear combination of orthogonal Chebyshev-Hermite polynomials

$$
P(u)=\sum_{k=0}^{p} \frac{C_{k}}{k !} H_{k}(u)
$$

clearly any polynomial $P(u)$ of degree less than or equal to $p$ admits a representation (4.2). Indeed,

$$
P(u)=\sum_{k=0}^{p} \frac{C_{k}}{k !} H_{k}(u), \quad C_{k}=\int_{\mathbb{R}} P(u) \varphi(u) H_{k}(u) \mathrm{d} u,
$$

where

$$
\varphi(u)=\frac{1}{\sqrt{2 \pi}} \mathrm{e}^{-u^{2} / 2}, \quad u \in \mathbb{R}
$$

is the standard Gaussian density. Without loss of generality, we can assume that $C_{p} \neq 0$ and $C_{0}=0$. Recall that the Chebyshev-Hermite polynomials have the representation

$$
H_{k}(u)=(-1)^{k}[\varphi(u)]^{-1} \frac{\mathrm{d}^{k}}{\mathrm{~d} u^{k}} \varphi(u), \quad k=0,1,2, \ldots
$$

We now use some ideas from Dobrushin and Major (1979) and Taqqu (1979). 
The random field $P(X(t, x ; \gamma))$ can be expanded in $\mathcal{L}_{2}(\Omega)$, the Hilbert space of random variables with finite second moments, as the orthogonal sum (see Major (1981) for details)

$$
P(X(t, x ; \gamma))=\sum_{k=1}^{p} \frac{C_{k}}{k !} I_{k}(t, x ; \gamma)
$$

where, for $v>1$,

$$
\begin{aligned}
I_{k}(t, x ; \gamma)= & \int_{\mathbb{R}^{2 k}}^{\prime} \exp \left[\mathrm{i}\left(\mu_{1}+\cdots+\mu_{k}\right) t+\mathrm{i}\left(\lambda_{1}+\cdots+\lambda_{k}\right) x\right] \\
& \times \prod_{j=1}^{k} f_{\gamma, \nu}^{1 / 2}\left(\mu_{j}, \lambda_{j}\right) \prod_{j=1}^{k} Z_{G}\left(\mathrm{~d} \mu_{j}, \mathrm{~d} \lambda_{j}\right) .
\end{aligned}
$$

Here $\int_{\mathbb{R}^{2 k}}^{\prime} \cdots$ is a multiple Wiener-Itô integral with respect to a Gaussian white noise measure $Z_{G}$. For the definition and properties of these integrals, see, e.g. Taqqu (1979) and Major (1981). We should note that the diagonal hyperplanes $\lambda_{i}= \pm \lambda_{j}, i, j=1, \ldots, k, i \neq j$, are excluded from the domain of integration.

Now, for $\gamma>0$ and $v>1$, consider the rescaled random fields

$$
Y_{\epsilon}(t, x ; \gamma)=\frac{1}{\epsilon^{p(3 / 4-v)}} P\left(X\left(\frac{t}{\epsilon}, \frac{x}{\sqrt{\epsilon}} ; \gamma \epsilon\right)\right)=\frac{C_{p}}{p !} X_{p}(t, x ; \gamma)+R_{\epsilon},
$$

where, by the Itô formula (see Major (1981, p. 30)), we have

$$
\begin{aligned}
X_{p}(t, x ; \gamma)= & \frac{1}{\epsilon^{p(3 / 4-v)}} H_{p}\left(X\left(\frac{t}{\epsilon}, \frac{x}{\sqrt{\epsilon}} ; \epsilon \gamma\right)\right) \\
= & \frac{1}{\epsilon^{p(3 / 4-v)}} \int_{\mathbb{R}^{2 p}}^{\prime} \exp \left[\mathrm{i}\left(\mu_{1}+\cdots+\mu_{p}\right) \frac{t}{\epsilon}+\mathrm{i}\left(\lambda_{1}+\cdots+\lambda_{p}\right) \frac{x}{\sqrt{\epsilon}}\right] \\
& \times \prod_{j=1}^{p} \frac{\sigma^{2}}{2 \pi} \frac{1}{\left(\mu_{j}^{2}+\left(\epsilon \gamma+c^{2} \lambda_{j}^{2}\right)^{2}\right)^{v / 2}} \prod_{j=1}^{p} Z_{G}\left(\mathrm{~d} \mu_{j}, \mathrm{~d} \lambda_{j}\right) \\
= & \int_{\mathbb{R}^{2 p}}^{\prime} \exp \left[\mathrm{i}\left(\mu_{1}+\cdots+\mu_{p}\right) t+\mathrm{i}\left(\lambda_{1}+\cdots+\lambda_{p}\right) x\right] \\
& \times \prod_{j=1}^{p} \frac{\sigma^{2}}{2 \pi} \frac{1}{\left(\mu_{j}^{2}+\left(\gamma+c^{2} \lambda_{j}^{2}\right)^{2}\right)^{v / 2}} \prod_{j=1}^{p} Z_{G}\left(\mathrm{~d} \mu_{j}, \mathrm{~d} \lambda_{j}\right) .
\end{aligned}
$$

Note that

$$
\operatorname{var} R_{\epsilon}=\operatorname{var} \sum_{k=1}^{p-1} \frac{C_{k}}{k !} \xi_{k, \epsilon} \leq K \sum_{k=1}^{p-1} \frac{C_{k}^{2}}{k !} \epsilon^{2 v(p-k)}
$$

where $K>0$ and $\xi_{k, \epsilon}=\left(\epsilon^{p(3 / 4-\nu)}\right)^{-1} H_{k}\left(X\left(t / \epsilon, x / \epsilon^{1 / 2} ; \epsilon \gamma\right)\right)$.

Thus, $R_{\epsilon} \rightarrow 0$ in probability as $\epsilon \rightarrow 0$. 
Note that the random field (4.5) is Gaussian if $p=1$ and non-Gaussian if $p \geq 2$, and that

$$
\begin{aligned}
\lim _{\epsilon \rightarrow 0} \operatorname{cov}\left(Y_{\epsilon}(t, x ; \gamma), Y_{\epsilon}\left(t^{\prime}, x^{\prime} ; \gamma\right)\right) \\
=\frac{C_{p}^{2}}{p !} \int_{\mathbb{R}^{2 p}} \exp \left[\mathrm{i}\left(\mu_{1}+\cdots+\mu_{p}\right)\left(t-t^{\prime}\right)+\mathrm{i}\left(\lambda_{1}+\cdots+\lambda_{p}\right)\left(x-x^{\prime}\right)\right] \\
\quad \times \prod_{j=1}^{p} \frac{\sigma^{2}}{2 \pi} \frac{1}{\left(\mu_{j}^{2}+\left(\gamma+c^{2} \lambda_{j}^{2}\right)^{2}\right)^{v}} \prod_{j=1}^{p} \mathrm{~d} \mu_{j} \mathrm{~d} \lambda_{j} \\
=\frac{C_{p}^{2}}{p !} \prod_{j=1}^{p} \int_{\mathbb{R}^{2}} \exp \left[\mathrm{i} \mu_{j}\left(t-t^{\prime}\right)+\mathrm{i} \lambda_{j}\left(x-x^{\prime}\right)\right] \frac{\sigma^{2}}{2 \pi} \frac{\mathrm{d} \mu_{j} \mathrm{~d} \lambda_{j}}{\left(\mu_{j}^{2}+\left(\gamma+c^{2} \lambda_{j}^{2}\right)^{2}\right)^{\nu}} .
\end{aligned}
$$

From (4.1)-(4.5) and Slutsky's arguments (see, e.g. Prakasa Rao (1987, p. 10)), we arrive at the following result.

Theorem 4.1. For $v>1$ and $\gamma>0$, the finite-dimensional distributions of the random fields $Y_{\epsilon}(t, x ; \gamma)$ converge weakly to the finite-dimensional distributions of the random field $X_{p}(t, x ; \gamma)$ as $\epsilon \rightarrow 0$.

Let $C(\Pi), \Pi=[0, T] \times[0, A]$, be the space of continuous functions on $\Pi$ with uniform topology, and let $\mathcal{P}_{\epsilon}, \epsilon>0$, be probability measures induced in $C(\Pi)$ by the fields $Y_{\epsilon}(t, x ; \gamma)$, $(t, x) \in \Pi$, defined in (4.4), and $\mathcal{P}$ be a probability measure induced in the space $C(\Pi)$ by the random field $X_{p}(t, x ; \gamma),(t, x) \in \Pi$, given in (4.5).

The following condition gives a way to prove the weak convergence of probability measures, denoted $\mathcal{P}_{\epsilon} \stackrel{\mathrm{W}}{\rightarrow} \mathcal{P}$, as $\epsilon \rightarrow 0$, in the space $C(\Pi)$ with uniform topology (see, e.g. Gīhman and Skorokhod (1971, p. 406)).

Lemma 4.1. If $v>v^{*}=2+\frac{1}{2}\left(\beta_{1}+\beta_{2}\right)$ for some $\beta_{1} \in(0,1], \beta_{2} \in(0,1]$, then

$$
J_{\beta_{1}, \beta_{2}, v, k}:=\int_{\mathbb{R}^{2 k}} \frac{\left|\lambda_{1}+\cdots+\lambda_{k}\right|^{1+\beta_{1}}\left|\mu_{1}+\cdots+\mu_{k}\right|^{1+\beta_{2}}}{\prod_{j=1}^{k}\left(\mu_{j}^{2}+\left(\gamma+c^{2} \lambda_{j}^{2}\right)^{2}\right)^{v}} \prod_{j=1}^{k} \mathrm{~d} \mu_{j} \mathrm{~d} \lambda_{j}<\infty
$$

for $1 \leq k \leq p$.

Proof. Using the inequalities

$$
\left|\lambda_{1}+\cdots+\lambda_{k}\right|^{1+\beta} \leq k^{1+\beta}\left(\max _{i}\left|\lambda_{i}\right|\right)^{1+\beta} \leq k^{1+\beta}\left(\left|\lambda_{1}\right|^{1+\beta}+\cdots+\left|\lambda_{k}\right|^{1+\beta}\right)
$$

and similar inequalities for $\mu_{1}, \ldots, \mu_{k}$ (replacing $\lambda_{1}, \ldots, \lambda_{k}$, respectively), we find that

$$
J_{\beta_{1}, \beta_{2}, v, k} \leq C\left(\beta_{1}, \beta_{2}, k\right)\left(J_{\beta_{1}, \beta_{2}, v, 1}\right)^{k} .
$$

Theorem 4.2. For $\gamma>0$ and $v>v^{*}$, the random field $X_{p}(t, x ; \gamma),(t, x) \in \Pi$, is almost surely continuous. Moreover, $\mathcal{P}_{\epsilon} \stackrel{\mathrm{w}}{\rightarrow} \mathcal{P}$ in the space $C(\Pi)$ with uniform topology as $\epsilon \rightarrow 0$.

Before proving Theorem 4.2, we state some known results (see, e.g. Chentsov (1960), Korolyuk et al. (1978) and Ivanov and Leonenko (1989, p. 8)).

Lemma 4.2. Let the finite-dimensional distributions of the random fields $Y_{\epsilon}(t, x),(t, x) \in \Pi$, converge to the finite-dimensional distributions of the field $Y(t, x),(t, x) \in \Pi$, which has continuous paths, and assume that the following conditions hold. 
(i) For all $\tau \geq 0$ and $h \geq 0$ such that $0 \leq \tau \leq T$ and $0 \leq h \leq A$, there exists a constant $K_{1}>0$ such that

$$
\mathrm{E}\left|Y_{\epsilon}(t+\tau, x+h)-Y_{\epsilon}(t, x+h)-Y_{\epsilon}(t+\tau, x)+Y_{\epsilon}(t, x)\right|^{p} \leq K_{1}(|\tau| \cdot|h|)^{1+q},
$$

where $p>0$ and $q>0$.

(ii) For all $\tau \in[0, T]$ and $x \in[0, A]$, there exist constants $K_{2}>0$ and $K_{3}>0$ such that

$$
\begin{aligned}
& \mathrm{E}\left|Y_{\epsilon}(t+\tau, x)+Y_{\epsilon}(t, x)\right|^{p} \leq K_{2}(|\tau|)^{1+q}, \\
& \mathrm{E}\left|Y_{\epsilon}(t, x+h)-Y_{\epsilon}(t, x)\right|^{p} \leq K_{3}(|h|)^{1+q},
\end{aligned}
$$

where $p>0$ and $q>0$.

Then $\mathcal{P}_{\epsilon} \stackrel{\mathrm{W}}{\rightarrow} \mathcal{P}$ as $\epsilon \rightarrow 0$, in the space $C(\Pi)$ with uniform topology, where $\mathcal{P}_{\epsilon}$ and $\mathcal{P}$ are probability measures induced by $Y_{\epsilon}$ and $Y$, respectively.

Lemma 4.3. Let assumptions (4.7), (4.8), and (4.9) hold when the random field $Y_{\epsilon}(t, x)$ is replaced by $Y(t, x),(t, x) \in \Pi$. Then the random field $Y(t, x),(t, x) \in \Pi$, induces $a$ probability measure in the space $C(\Pi)$ with uniform topology.

Slightly different conditions on the existence of continuous modifications of random fields are given in Ibragimov (1983) (see also Ibragimov and Khasminskii (1981)).

Proof of Theorem 4.2. From the condition $v>v^{*}$, we obtain

$$
\begin{aligned}
\mathrm{E} \mid X_{p}(t+\tau, x+h)- & X_{p}(t, x+h)-X_{p}(t+\tau, x)+\left.X_{p}(t, x)\right|^{2} \\
\leq \frac{\sigma^{2 p}}{(2 \pi)^{2 p}} \frac{C_{p}^{2}}{p !} \int_{\mathbb{R}^{2 p}} \mid & \exp \left[\mathrm{i}\left(\mu_{1}+\cdots+\mu_{p}\right)(t+\tau)+\mathrm{i}\left(\lambda_{1}+\cdots+\lambda_{p}\right)(x+h)\right] \\
& -\exp \left[\mathrm{i}\left(\mu_{1}+\cdots+\mu_{p}\right) t+\mathrm{i}\left(\lambda_{1}+\cdots+\lambda_{p}\right)(x+h)\right] \\
& -\exp \left[\mathrm{i}\left(\mu_{1}+\cdots+\mu_{p}\right)(t+\tau)+\mathrm{i}\left(\lambda_{1}+\cdots+\lambda_{p}\right) x\right] \\
& +\left.\exp \left[\mathrm{i}\left(\mu_{1}+\cdots+\mu_{p}\right) t+\mathrm{i}\left(\lambda_{1}+\cdots+\lambda_{p}\right) x\right]\right|^{2} \\
& \times \prod_{j=1}^{p} \frac{1}{\left(\mu_{j}^{2}+\left(\gamma+c^{2} \lambda_{j}^{2}\right)^{2}\right)^{v}} \prod_{j=1}^{p} \mathrm{~d} \mu_{j} \mathrm{~d} \lambda_{j}
\end{aligned}
$$

where $J_{\beta_{1}, \beta_{2}, v, p}$ is as defined in (4.6).

Thus, (4.7) holds for the random field $X_{m}(t, x),(t, x) \in \Pi$. Similarly, we can check conditions (4.8) and (4.9). By Lemma 4.3, the random field $X_{m}(t, x),(t, x) \in \Pi$ induces the probability measure in the space $C(\Pi)$ with uniform topology. 
For the random field $Y_{\epsilon}(t, x),(t, x) \in \Pi$, we obtain

$$
\begin{aligned}
& \mathrm{E}\left|Y_{\epsilon}(t+\tau, x+h)-Y_{\epsilon}(t, x+h)-Y_{\epsilon}(t+\tau, x)+Y_{\epsilon}(t, x)\right|^{2} \\
& \leq \frac{1}{\epsilon^{2 p(3 / 4-\nu)}} \sum_{k=1}^{p} \frac{C_{k}^{2}}{k !} \frac{\sigma^{2 k}}{(2 \pi)^{2 k}} \mathrm{E}\left[I_{k}\left(\frac{t+\tau}{\epsilon}, \frac{x+h}{\sqrt{\epsilon}} x ; \gamma \epsilon\right)-I_{k}\left(\frac{t}{\epsilon}, \frac{x+h}{\sqrt{\epsilon}} x ; \gamma \epsilon\right)\right. \\
& \left.-I_{k}\left(\frac{t+\tau}{\epsilon}, \frac{x}{\sqrt{\epsilon}} x ; \gamma \epsilon\right)+I_{k}\left(\frac{t}{\epsilon}, \frac{x}{\sqrt{\epsilon}} x ; \gamma \epsilon\right)\right]^{2} \\
& \leq \sum_{k=1}^{p} \frac{C_{k}^{2}}{k !} \frac{\sigma^{2 k}}{(2 \pi)^{2 k}} \epsilon^{2(p-k)(\nu-3 / 4)} \int_{\mathbb{R}^{2 k}} 4 \sin ^{2}\left[\frac{1}{2}\left(\lambda_{1}+\cdots+\lambda_{k}\right) h\right] 4 \sin ^{2}\left[\frac{1}{2}\left(\mu_{1}+\cdots+\mu_{k}\right) \tau\right] \\
& \times \prod_{j=1}^{k} \frac{1}{\left(\mu_{j}^{2}+\left(\gamma+c^{2} \lambda_{j}^{2}\right)^{2}\right)^{v}} \prod_{j=1}^{k} \mathrm{~d} \mu_{j} \mathrm{~d} \lambda_{j} \\
& \leq(|\tau| \cdot|h|)^{1+\min \left\{\beta_{1}, \beta_{2}\right\}} \sum_{k=1}^{p} \frac{C_{k}^{2}}{k !} \frac{\sigma^{2 k}}{(2 \pi)^{2 k}} \epsilon^{2(p-k)(\nu-3 / 4)} J_{\beta_{1}, \beta_{2}, v, k} \\
& \leq Q_{\epsilon} K_{1}(|\tau| \cdot|h|)^{1+\min \left\{\beta_{1}, \beta_{2}\right\}},
\end{aligned}
$$

where

$$
Q_{\epsilon}=\sum_{k=1}^{p} \epsilon^{2(p-k)(v-3 / 4)} \int_{\mathbb{R}^{2 k}} \frac{\left|\lambda_{1}+\cdots+\lambda_{k}\right|^{1+\beta_{1}}\left|\mu_{1}+\cdots+\mu_{k}\right|^{1+\beta_{2}}}{\prod_{j=1}^{k}\left(\mu_{j}^{2}+\left(\gamma^{2}+c^{2} \lambda_{j}^{2}\right)^{2}\right)^{v}} \prod_{j=1}^{k} d \lambda_{j} d \mu_{j},
$$

$K_{1}$ is a positive constant and, by Lemma 4.1, we have

$$
\lim _{\epsilon \rightarrow 0} Q_{\epsilon}=J_{\beta_{1}, \beta_{2}, v, p}<\infty .
$$

Thus, the bounds (4.7), (4.8), and (4.9) all hold and the family of measures $\mathcal{P}_{\epsilon}$ is compact in $C(\Pi)$ with uniform topology. The last statement of Theorem 4.2 follows from Theorem 4.1 and Lemmas 4.2 and 4.3.

\section{Appendix A.}

Auxiliary definitions and results needed in the derivation of a formal second-order solution to (2.1) and (3.1) are now provided. Specifically, the main elements of the theory of generalized random fields on fractional Sobolev spaces are briefly described (see Ruiz-Medina et al. (2001), (2004)).

Let $C_{0}^{\infty}\left(\mathbb{R}^{d}\right)$ be the space of infinitely differentiable functions with compact support contained in $\mathbb{R}^{d}$, and let $\delta\left(\mathbb{R}^{d}\right)$ be the space of $C^{\infty}$ functions with rapid decay at infinity. The duals of these spaces are respectively known as the space of distributions $\left[C_{0}^{\infty}\left(\mathbb{R}^{d}\right)\right]^{\prime}$ and the space of tempered distributions $f^{\prime}\left(\mathbb{R}^{d}\right)$, with

$$
C_{0}^{\infty}\left(\mathbb{R}^{d}\right) \subseteq s\left(\mathbb{R}^{d}\right) \subseteq \varsigma^{\prime}\left(\mathbb{R}^{d}\right) \subseteq\left[C_{0}^{\infty}\left(\mathbb{R}^{d}\right)\right]^{\prime} .
$$

Fractional Sobolev spaces on $\mathbb{R}^{d}$ are introduced as spaces of tempered distributions with positive or negative weak-sense fractional orders of differentiation (weak-sense regularity) and integration (singularity). We denote by $H^{s}\left(\mathbb{R}^{d}\right)$ the fractional Sobolev space of order $s$ on $\mathbb{R}^{d}$, and by $H^{-s}\left(\mathbb{R}^{d}\right)$ its dual Hilbert space (see Triebel (1978)). 
Definition A.1. For $\alpha \in \mathbb{R}$, a random function $X_{\alpha}$ from $H^{\alpha}\left(\mathbb{R}^{d}\right)$ into $\mathcal{L}^{2}(\Omega) \equiv \mathcal{L}^{2}(\Omega, \mathcal{A}, \mathrm{P})$ is said to be an $\alpha$-generalized random field ( $\alpha$-GRF) if it is linear and continuous in the meansquare sense with respect to the $H^{\alpha}$ topology. Here, $\mathcal{L}^{2}(\Omega, \mathcal{A}, \mathrm{P})$ denotes the Hilbert space of zero-mean random variables defined on the basic complete probability space $(\Omega, \mathcal{A}, \mathrm{P})$ with finite second-order moments and with the inner product

$$
\langle X, Y\rangle_{\mathcal{L}^{2}(\Omega)}=\mathrm{E}[X \bar{Y}], \quad X, Y \in \mathcal{L}^{2}(\Omega) .
$$

For an $\alpha$-GRF, $\alpha$ denotes its fractional singularity order and $-\alpha$ its fractional regularity order. For $\alpha \geq 0, X_{\alpha}$ is a random distribution in the mean-square sense and, for $\alpha<0, X_{\alpha}$ defines an ordinary random field $\mathcal{X}_{\alpha}$ - in the weak sense for $-\alpha \leq \frac{1}{2} d$ and in the strong sense for $-\alpha>\frac{1}{2} d-$ in terms of the mean-square integral

$$
X_{\alpha}(\phi) \stackrel{\text { m.s. }}{=} \int_{\mathbb{R}^{d}} \chi_{\alpha}(z) \bar{\phi}(z) \mathrm{d} z \quad \text { for all } \phi \in H^{\alpha}\left(\mathbb{R}^{d}\right),
$$

where $\mathcal{X}_{\alpha}$ is continuous in the mean-square sense for $-\alpha>\frac{1}{2} d$.

From the kernel theorem (Gel'fand and Vilenkin (1964)), the covariance function $B_{X_{\alpha}}$ of $X_{\alpha}$ admits the representation

$$
B_{X_{\alpha}}(\varphi, \phi)=\left\langle\left(R_{X_{\alpha}} \varphi\right)^{*}, \phi\right\rangle_{H^{\alpha}\left(\mathbb{R}^{d}\right)}, \quad \varphi, \phi \in H^{\alpha}\left(\mathbb{R}^{d}\right),
$$

since $B_{X_{\alpha}}$ defines a bilinear continuous form on $H^{\alpha}\left(\mathbb{R}^{d}\right)$. Here, '*, stands for the duality between Hilbert spaces (Riesz representation theorem), and $R_{X_{\alpha}}$ is the covariance operator of $X_{\alpha}$, that is, a symmetric, positive, continuous linear operator from $H^{\alpha}\left(\mathbb{R}^{d}\right)$ into $H^{-\alpha}\left(\mathbb{R}^{d}\right)$. Hence, for $-\alpha>\frac{1}{2} d, R_{X_{\alpha}}$ admits an integral representation in terms of a continuous kernel $B x_{\alpha}$, the covariance function of $\mathcal{X}_{\alpha}$.

Definition A.2. For $\alpha \in \mathbb{R}$, we say that the fractional generalized random field (FGRF) $\widetilde{X}_{\alpha}: H^{-\alpha}\left(\mathbb{R}^{d}\right) \rightarrow \mathcal{L}^{2}(\Omega, \mathcal{A}, \mathrm{P})$ is the ' $\alpha$-dual' of the $\alpha$-GRF $X_{\alpha}: H^{\alpha}\left(\mathbb{R}^{d}\right) \rightarrow \mathcal{L}^{2}(\Omega, \mathcal{A}, \mathrm{P})$ if it satisfies

(i) $H\left(X_{\alpha}\right)=H\left(\tilde{X}_{\alpha}\right)$,

(ii) $\left\langle X_{\alpha}(\phi), \widetilde{X}_{\alpha}(g)\right\rangle_{H\left(X_{\alpha}\right)}=\left\langle\phi, g^{*}\right\rangle_{H^{\alpha}\left(\mathbb{R}^{d}\right)}$ for $\phi \in H^{\alpha}\left(\mathbb{R}^{d}\right)$ and $g \in H^{-\alpha}\left(\mathbb{R}^{d}\right)$, with $g^{*}$ being the dual element of $g$ with respect to the $H^{\alpha}$ topology.

Let $H\left(X_{\alpha}\right)$ be the Hilbert space of random variables associated with $X_{\alpha}$, that is, the Hilbert space of random variables defined as the closed span in the $\mathcal{L}^{2}(\Omega)$ topology of $\left\{X_{\alpha}(\varphi): \varphi \in\right.$ $\left.H^{\alpha}\left(\mathbb{R}^{d}\right)\right\}$. Also, let $\mathcal{H}\left(X_{\alpha}\right)$ be the RKHS defined by the covariance function of $X_{\alpha}$, that is, the subspace of functions $f$ of $H^{-\alpha}\left(\mathbb{R}^{d}\right)$ given by

$$
\left\{f \in H^{-\alpha}\left(\mathbb{R}^{d}\right): f(\varphi)=\mathrm{E}\left[X \bar{X}_{\alpha}(\varphi)\right] \text { for all } \varphi \in H^{\alpha}\left(\mathbb{R}^{d}\right)\right\},
$$

for a certain $X \in H\left(X_{\alpha}\right)$. Similarly, let $H\left(\tilde{\widetilde{X}}_{\alpha}\right)$ and $\mathcal{H}\left(\widetilde{X}_{\alpha}\right)$ respectively represent the Hilbert space of random variables associated with $\widetilde{X}_{\alpha}$ and the RKHS associated with $\widetilde{X}_{\alpha}$.

The following result shows that the RKHS of an $\alpha$-GRF $X_{\alpha}$ having dual $\tilde{X}_{\alpha}$ has norm equivalent to the norm of $H^{-\alpha}\left(\mathbb{R}^{d}\right)$. The covariance factorization of $X_{\alpha}$ is then obtained in terms of isomorphisms, and a white noise linear filter representation of $X_{\alpha}$ derived in terms of isomorphisms (Ruiz-Medina et al. (2001), (2004)). 
Proposition A.1. Under the duality condition, the following assertions hold.

(i) For $\alpha \in \mathbb{R}$, the identity operator $i$ between the Hilbert spaces $\mathcal{H}\left(X_{\alpha}\right)$ and $H^{-\alpha}\left(\mathbb{R}^{d}\right)$ (and $\tilde{l}$ between the spaces $\mathscr{H}\left(\widetilde{X}_{\alpha}\right)$ and $H^{\alpha}\left(\mathbb{R}^{d}\right)$ ) defines an isomorphism. That is, the norms defined on these spaces are equivalent.

(ii) $S_{\alpha}^{-1}=(i J)^{-1}=\widetilde{X}_{\alpha}, \widetilde{S}_{\alpha}^{-1}=(i \widetilde{J})^{-1}=X_{\alpha}, R_{X_{\alpha}}=S_{\alpha} \widetilde{S}_{\alpha}^{-1}$, and $R_{\tilde{X}_{\alpha}}=\widetilde{S}_{\alpha} S_{\alpha}^{-1}$, where $J$ represents the isometric isomorphism relating $H\left(X_{\alpha}\right)$ to $\mathcal{H}\left(X_{\alpha}\right)$, and $\widetilde{J}=\left(J^{-1}\right)^{\dagger}$ the isometric isomorphism relating $H\left(\widetilde{X}_{\alpha}\right)$ to $\mathcal{H}\left(\tilde{X}_{\alpha}\right)$. (Here, '†' stands for the adjoint operator.) The operators $R_{X_{\alpha}}$ and $R_{\tilde{X}_{\alpha}}$ represent, as before, the respective covariance operators of $X_{\alpha}$ and $\tilde{X}_{\alpha}$. Note that $S_{\alpha}^{-1}=\tilde{X}_{\alpha}$ means that

$$
J \tilde{X}_{\alpha}(f)(\phi)=f(\phi) \text { for all } \phi \in H^{\alpha}\left(\mathbb{R}^{d}\right)
$$

and for each $f \in H^{-\alpha}\left(\mathbb{R}^{d}\right)$, and that

$$
\tilde{X}_{\alpha} J X \stackrel{H\left(X_{\alpha}\right)}{=} X \quad \text { for all } X \in H\left(X_{\alpha}\right) .
$$

The relation $\widetilde{S}_{\alpha}^{-1}=X_{\alpha}$ is understood similarly, in terms of the operator $\widetilde{J}$.

(iii) The FGRFs $X_{\alpha}$ and $\tilde{X}_{\alpha}$ satisfy

$$
\begin{gathered}
\left.X_{\alpha}\left(\widetilde{S}_{\alpha} S_{\varepsilon}^{-1} h\right)\right) \stackrel{\mathcal{L}^{2}(\Omega)}{=} \varepsilon(h) \quad \text { for all } h \in L^{2}\left(\mathbb{R}^{d}\right) \text { and } \\
\tilde{X}_{\alpha}\left(S_{\alpha} S_{\varepsilon}^{-1} g\right) \stackrel{\mathcal{L}^{2}(\Omega)}{=} \varepsilon(g) \quad \text { for all } g \in L^{2}\left(\mathbb{R}^{d}\right),
\end{gathered}
$$

where $\varepsilon$ represents generalized white noise. That is, $\varepsilon$ is an $\alpha-G R F$ with $\alpha=0$ and with covariance function

$$
B_{\varepsilon}(h, v)=\mathrm{E}[\varepsilon(h) \varepsilon(v)]=\langle h, v\rangle_{L^{2}\left(\mathbb{R}^{d}\right)} \text { for all } h, v \in L^{2}\left(\mathbb{R}^{d}\right) .
$$

Here, $S_{\varepsilon}=J_{\varepsilon}$ is the isometric isomorphism between the space $H(\varepsilon)$, generated by $\varepsilon$, and $L^{2}\left(\mathbb{R}^{d}\right)=\mathscr{H}(\varepsilon)$.

(iv) For $-\alpha>\frac{1}{2} d, H^{-\alpha}\left(\mathbb{R}^{d}\right)=\mathscr{H}\left(X_{\alpha}\right)$ is continuously embedded into $\mathcal{C}^{-\alpha-d / 2}\left(\mathbb{R}^{d}\right)$. The random field

$$
\mathcal{X}_{\alpha}(z) \stackrel{\text { m.s. }}{=} \int_{\mathbb{R}^{d}}\left[\int_{\mathbb{R}^{d}} \mathrm{i} \lambda^{1 / 2} \Phi(z, y ; \lambda) \mathrm{d} \rho(\lambda)\right] \mathrm{d} \varepsilon(y) \quad \text { for all } z \in \mathbb{R}^{d}
$$

then provides the unique mean-square, continuous, ordinary solution to (A.1), where $\Phi$ and $\rho$ respectively represent the spectral kernel and the spectral measure associated with the integral spectral representation of the covariance operator $R_{X_{\alpha}}$.

Similarly, for $\alpha>\frac{1}{2} d, H^{\alpha}\left(\mathbb{R}^{d}\right)=\mathscr{H}\left(\widetilde{X}_{\alpha}\right)$ is continuously embedded into $\mathcal{C}^{\alpha-d / 2}\left(\mathbb{R}^{d}\right)$, and (A.2) admits a unique mean-square, continuous, ordinary solution defined by

$$
\tilde{\mathcal{X}}_{\alpha}(z) \stackrel{\text { m.s. }}{=} \int_{\mathbb{R}^{d}}\left[\int_{\mathbb{R}^{d}} \tilde{\mathrm{i}}^{1 / 2} \widetilde{\Phi}(z, y ; \tilde{\lambda}) \mathrm{d} \widetilde{\rho}(\widetilde{\lambda})\right] \mathrm{d} \varepsilon(y) \quad \text { for all } z \in \mathbb{R}^{d},
$$

with $\widetilde{\Phi}$ and $\widetilde{\rho}$ respectively representing the spectral kernel and the spectral measure associated with the integral spectral representation of the covariance operator $R_{\widetilde{X}_{\alpha}}$. 


\section{Acknowledgements}

This work was partially supported by EPSRC grant RCMT091, project BFM2002-01836 of the DGI, Spain, and the Australian Research Council grants A10024117 and DP 0345577.

The constructive comments from a referee and from V. V. Anh and J. M. Angulo are gratefully acknowledged.

\section{References}

Adams, R. A. (1975). Sobolev Spaces. Academic Press, New York.

AdLer, R. J. (1981). The Geometry of Random Fields. John Wiley, New York.

Angulo, J. M., Ruiz-Medina, M. D. And ANh, V. V. (2000a). Estimation and filtering of fractional generalized random fields. J. Austral. Math. Soc. A 69, 336-361.

Angulo, J. M., Ruiz-Medina, M. D., Anh, V. V. And Grecksch, W. (2000b). Fractional diffusion and fractional heat equation. Adv. Appl. Prob. 32, 1077-1099.

ANH, V. V. AND LeonenKo, N. N. (2000). Scaling laws for fractional diffusion-wave equations with singular data. Statist. Prob. Lett. 48, 239-252.

AnH, V. V. And Leonenko, N. N. (2001). Spectral analysis of fractional kinetic equations with random data. J. Statist. Phys. 104, 1349-1487.

ANH, V. V. AND LEONEnKo, N. N. (2002). Renormalization and homogenization of fractional diffusion equations with random data. Prob. Theory Relat. Fields 124, 381-408.

Anh, V. V., Angulo, J. M. And Ruiz-Medina, M. D. (1999). Possible long-range dependence in fractional random fields. J. Statist. Planning Infer. 80, 95-110.

Chambers, M. J. (1996). The estimation of continuous parameter long-memory time series models. Econom. Theory 12, 373-390.

Chentsov, N. N. (1960). Limit theorems for certain classes of random functions. In Proc. All-Union Conf. Theory Prob. Math. Statist., Armenian Academy of Sciences, Yerevan, pp. 280-285 (in Russian).

Christakos, G. (1991). A theory of spatio-temporal random fields and its application to space-time data processing. IEEE Trans. Systems Man. Cybernet. 21, 861-875.

Christakos, G. (2000). Modern Spatiotemporal Geostatistics. Oxford University Press.

Dautray, R. And Lions, J. L. (1985a). Mathematical Analysis and Numerical Methods for Science and Technology, Vol. 2, Functional and Variational Methods. Springer, New York.

Dautray, R. And Lions, J. L. (1985b). Mathematical Analysis and Numerical Methods for Science and Technology, Vol. 3, Spectral Theory and Applications. Springer, New York.

Dobrushin, R. L. (1979). Gaussian and their subordinated self-similar random generalized fields. Ann. Prob. 7, 1-28.

Dobrushin, R. L. AND Major, P. (1979). Non-central limit theorem for non-linear functionals of Gaussian fields. Z. Wahrscheinlichkeitsth. 50, 1-28.

Donoghue, W. J. (1969). Distributions and Fourier Transforms. Academic Press, New York.

Dunford, N. AND Schwartz, J. T. (1971). Linear Operators. John Wiley, New York.

GAY, R. AND HeYDE, C. C. (1990). On a class of random field model which allows long dependence. Biometrika 77, 401-403.

Gel’́fand, I. M. and Vilenkin, N. Ya. (1964). Generalized Functions, Vol. 4. Academic Press, New York.

Gīhman, İ. İ. And Skorokhod, A. V. (1971). The Theory of Stochastic Processes, Vol. 1. Springer, Berlin.

Granger, C. W. J. And Joyeux, R. (1980). An introduction to long-memory time series models and fractional differencing. J. Time Series Anal. 10, 233-257.

Heyde, C. C. (1997). Quasi-Likelihood and Its Applications: A General Approach to Optimal Parameter Estimation. Springer, New York.

Hilfer, R. (ed.) (2000). Applications of Fractional Calculus in Physics. World Scientific, Singapore.

Hosking, J. R. M. (1981). Fractional differencing. Biometrika 68, 165-176.

Ibragimov, I. A. (1983). On smoothness conditions for trajectories of random functions. Theory Prob. Appl. 28, 240-262 (in Russian).

Ibragimov, I. A. And Khasminskit, R. Z. (1981). Statistical Estimation: Asymptotic Theory. Springer, New York.

Ivanov, A. B. AND LeOnenKo, N. N. (1989). Statistical Analysis of Random Fields. Kluwer, Dordrecht.

JoNEs, R. H. AND ZHANG, Y. (1997). Models for continuous stationary space-time processes. In Modelling Longitudinal and Spatially Corrected Data, eds T. G. Gregoire et al., Springer, New York, pp. 343-449.

Karatzas I. And Shreve S. E. (1991). Brownian Motion and Stochastic Calculus. Springer, New York.

Korolyuk, V. S., Portenko, N. I., Skorokhod, A. V. And Turbin, A. F. (1978). Handbook on Probability Theory and Mathematical Statistics. Naukova Dumka, Kiev (in Russian). 
Mainardi, F., Luchko, Y. And Pagnini, G. (2001). The fundamental solution of the space-time fractional diffusion equation. Fract. Calc. Appl. Anal. 4, 153-192.

Major, P. (1981). Multiple Wiener-Itô Integrals (Lecture Notes Math. 849). Springer, Berlin.

Prakasa Rao, B. L. S. (1987). Asymptotic Theory of Statistical Inference. John Wiley, New York.

Protter, P. (1990). Stochastic Integration and Differential Equations (Appl. Math. (New York) 21). Springer, Berlin.

RAmm, A. G. (1990). Random Fields Estimation Theory. Longman Scientific and Technical, London.

Ruiz-Medina, M. D., Angulo, J. M. And Anh, V. V. (2003). Fractional generalized random fields on bounded domains. Stoch. Anal. Appl. 21, 465-492.

Ruiz-Medina, M. D., Angulo, J. M. And Anh, V. V. (2004). Fractional random fields on domains with fractal boundary. Infinit. Dimens. Anal. Quantum Prob. Relat. Top. 7, 395-417.

Ruiz-Medina, M. D., Anh, V. V. And Angulo, J. M. (2001). Stochastic fractional-order differential models with fractal boundary conditions. Statist. Prob. Lett. 54, 47-60.

Stein, E. M. (1970). Singular Integrals and Differential Properties of Functions. Princeton University Press.

STEIN, M. L. (1999). Interpolation of Spatial Data. Springer, Berlin.

TAQQU, M. S. (1979). Convergence of integrated processes of arbitrary Hermite rank. Z. Wahrscheinlichkeitsth. 50, 53-83.

Triebel, H. (1978). Interpolation Theory, Function Spaces, Differential Operators. North-Holland, Amsterdam.

Whittle, P. (1954). On stationary processes in the plane. Biometrika 41, 434-449.

Whittle, P. (1963). Stochastic processes in several dimensions. Bull. Inst. Internat. Statist. 40, 974-994.

Wong, E. And HaJeK, B. (1985). Stochastic Processes in Engineering Systems. Springer, New York.

WOYCZYŃSKI, W. A. (1998). Burgers-KPZ Turbulence (Lecture Notes Math. 1700). Springer, Berlin.

YADRENKo, M. I. (1983). Spectral Theory of Random Fields. Optimization Software, New York. 